\title{
QUASISYMMETRIC SPHERES OVER JORDAN DOMAINS
}

\author{
VYRON VELLIS AND JANG-MEI WU
}

\begin{abstract}
Let $\Omega$ be a planar Jordan domain. We consider double-dome-like surfaces $\Sigma$ defined by graphs of functions of $\operatorname{dist}(\cdot, \partial \Omega)$ over $\Omega$. The goal is to find the right conditions on the geometry of the base $\Omega$ and the growth of the height so that $\Sigma$ is a quasisphere or is quasisymmetric to $\mathbb{S}^{2}$. An internal uniform chord-arc condition on the constant distance sets to $\partial \Omega$, coupled with a mild growth condition on the height, gives a close-to-sharp answer. Our method also produces new examples of quasispheres in $\mathbb{R}^{n}$, for any $n \geq 3$.
\end{abstract}

\section{INTRODUCTION}

Images of $\mathbb{S}^{n}$ under quasiconformal homeomorphisms of $\mathbb{R}^{n+1}$ are called quasispheres. In $\mathbb{R}^{2}$, quasicircles can be described completely in geometrical terms ([1], [10, [19]). In higher dimensions the only known characterization is due to Gehring [8] and Väisälä [21]: a topological $n$-sphere $\Sigma$ in $\mathbb{R}^{n+1}$ is a quasisphere if and only if the bounded component and the unbounded component of $\mathbb{R}^{n+1} \backslash \Sigma$ are quasiconformally equivalent to $\mathbb{B}^{n+1}$ and $\mathbb{R}^{n+1} \backslash \overline{\mathbb{B}^{n+1}}$, respectively. Intriguing examples of quasispheres have been constructed drawing ideas from harmonic analysis, conformal dynamics and classical geometric topology ([3], [5], [14, [15], [16]). The basic question of a geometric characterization of quasispheres remains.

More generally, metric spaces that are quasisymmetrically homeomorphic to $\mathbb{S}^{n}$ are called quasisymmetric spheres. An intricate characterization of quasisymmetric 2-spheres has been found by Bonk and Kleiner [4]; as a consequence, the Ahlfors 2-regularity together with the local linear connectivity on a metric 2-sphere suffices. However, little is known about quasisymmetric $n$-spheres when $n \geq 3$.

In this article, we examine double-dome-like surfaces in $\mathbb{R}^{3}$ defined by graphs of functions on a Jordan domain $\Omega \subset \mathbb{R}^{2}$,

$$
\Sigma(\Omega, \varphi)=\{(x, z): x \in \bar{\Omega}, z= \pm \varphi(\operatorname{dist}(x, \partial \Omega))\}
$$

where $\varphi(\cdot)$ is a continuous increasing function on $[0, \infty)$ with $\varphi(0)=0$. Our aim is to find the right conditions on the geometry of the base $\Omega$ and the growth of the gauge $\varphi$ in order for these surfaces to be quasispheres or quasisymmetric spheres.

Suppose that $\Omega$ is a planar Jordan domain and $\mathbb{B}^{3}$ is the open unit ball. Gehring [9] showed that the slit domain $\mathbb{R}^{3} \backslash \bar{\Omega}$ is quasiconformally homeomorphic to $\mathbb{R}^{3} \backslash \overline{\mathbb{B}^{3}}$ if and only if $\Omega$ is a quasidisk; Väisälä proved $[23$. that the infinite cylinder $\Omega \times \mathbb{R}$ is quasiconformally equivalent to $\mathbb{B}^{3}$ if and only if $\Omega$ satisfies an internal chord arc condition. A slit domain may be regarded as the complement of $\Sigma(\Omega, \varphi)$ when

Received by the editors July 17, 2014.

2010 Mathematics Subject Classification. Primary 30C65; Secondary 30C62.

Key words and phrases. Quasispheres, quasisymmetric spheres, double-dome-like surfaces, level chord-arc property.

This research was supported in part by the NSF grant DMS-1001669. 
$\varphi \equiv 0$ in the previous setting, and a cylindrical domain may be regarded as the domain enclosed by $\Sigma(\Omega, \varphi)$ by choosing $\varphi \equiv \infty$. In this spirit and for $\varphi(t)=t$, we have the following.

Theorem 1.1. Suppose that $\Omega$ is a Jordan domain in $\mathbb{R}^{2}$. Then the surface

$$
\Sigma(\Omega, t)=\{(x, z): x \in \bar{\Omega}, z= \pm \operatorname{dist}(x, \partial \Omega)\}
$$

is a quasisphere if and only if $\partial \Omega$ is a quasicircle.

The method of constructing quasispheres in Theorem 1.1 extends inductively to all dimensions; see Theorem 4.6 .

For $\alpha>1$, surfaces $\Sigma\left(\Omega, t^{\alpha}\right)=\left\{(x, z): x \in \bar{\Omega}, z= \pm(\operatorname{dist}(x, \partial \Omega))^{\alpha}\right\}$ are not linearly locally connected, therefore are not quasisymmetric spheres.

Väisälä 22] has shown that the product $\gamma \times I$ of a Jordan $\operatorname{arc} \gamma$ and an interval $I$ is quasisymmetric embeddable in $\mathbb{R}^{2}$ if and only if $\Gamma$ satisfies the chord-arc condition.

When $0<\alpha<1$, the part of the surface $\Sigma\left(\Omega, t^{\alpha}\right)$ near $\mathbb{R}^{2} \times\{0\}$ resembles the product of an arc with an interval locally. On the other hand, the double-dome-like surface envelops the interior $\Omega$ above and below. Therefore some form of internal uniform chord-arc condition is expected in order for $\Sigma\left(\Omega, t^{\alpha}\right)$ to be quasisymmetric to $\mathbb{S}^{2}$.

A Jordan domain $\Omega$ is said to have the level chord-arc property if the $\epsilon$-distance sets to $\partial \Omega$,

$$
\gamma_{\epsilon}=\{x \in \Omega: \operatorname{dist}(x, \partial \Omega)=\epsilon\}
$$

are uniform $c$-chord-arc curves for some $c>1$ and all sufficiently small $\epsilon>0$. We define a class of gauges

$$
\begin{aligned}
\mathcal{F}=\{\varphi:[0, \infty) \rightarrow[0, \infty): \text { a homeomorphism with } \varphi(0)=0, \\
\left.\quad \liminf _{t \rightarrow 0} \varphi(t) / t>0 \text { and } \varphi \text { is Lipschitz on }[r,+\infty) \text { for all } r>0\right\} .
\end{aligned}
$$

This class includes $t^{\alpha}$ with $0<\alpha<1$. We now state the main theorem.

Theorem 1.2. Let $\Omega$ be a planar Jordan domain. Then the surface $\Sigma(\Omega, \varphi)$ is quasisymmetric to $\mathbb{S}^{2}$ for every $\varphi$ in $\mathcal{F}$ if and only if $\Omega$ has the level chord-arc property.

In general, a Lipschitz domain may contain a sequence $\gamma_{\epsilon_{n}}$ of constant distance sets with $\epsilon_{n} \rightarrow 0$, each of which is a Jordan curve containing a cusp, hence not a quasicircle; see Remark 5.2 in [24].

What are the intrinsic characteristics of a Jordan domain that has the level chord-arc property? A flatness module $\zeta_{\gamma}$ measuring the deviation of subarcs of a Jordan curve $\gamma$ from their chords in a uniform and scale-invariant way has been defined in 24] for this purpose.

From here onward, given $x, y$ on a Jordan curve $\gamma$, we take $\gamma(x, y)$ to be the subarc of $\gamma$ connecting $x$ and $y$ of smaller diameter or either subarc when both have the same diameter; given $x, y$ in a Euclidean space, we denote by $l_{x, y}$ the infinite line containing $x$ and $y$. Set

$$
\zeta_{\gamma}(x, y)=\frac{1}{|x-y|} \sup _{z \in \gamma(x, y)} \operatorname{dist}\left(z, l_{x, y}\right)
$$

and define the flatness of $\gamma$ to be

$$
\zeta_{\gamma}=\lim _{r \rightarrow 0} \sup _{x, y \in \gamma,|x-y| \leq r} \zeta_{\gamma}(x, y) .
$$


The connection between the flatness of $\gamma$ and the level chord-arc property has been established in [24].

Theorem 1.3 (24, Theorem 1.2, Theorem 1.3]). Let $\Omega$ be a planar Jordan domain and suppose that $\partial \Omega$ is a chord-arc curve with flatness $\zeta_{\partial \Omega}<1 / 2$. Then $\Omega$ has the level chord-arc property. On the other hand, there exists a Jordan domain $\Omega$ whose boundary $\partial \Omega$ is a chord-arc with flatness $\zeta_{\partial \Omega}=1 / 2$ which does not satisfy the level chord-arc property.

Combining Theorem 1.2 and Theorem 1.3 with Lemma 5.6 below, we may obtain the following.

Corollary 1.4. Suppose that $\Omega$ is a planar Jordan domain whose boundary is a chord-arc curve with flatness $\zeta_{\partial \Omega}<1 / 2$. Then the surface $\Sigma\left(\Omega, t^{\alpha}\right)$ is quasisymmetric to $\mathbb{S}^{2}$ for all $\alpha \in(0,1]$. On the other hand, there exist Jordan domains $\Omega$ in $\mathbb{R}^{2}$ whose boundaries are chord-arc curves with flatness $\zeta_{\partial \Omega}=1 / 2$ to which the associated surfaces $\Sigma\left(\Omega, t^{\alpha}\right)$ are not quasisymmetric to $\mathbb{S}^{2}$ for any $\alpha \in(0,1)$.

The growth condition near 0 imposed on the gauges in $\mathcal{F}$ is essential. In fact, if $\liminf _{t \rightarrow 0} \varphi(t) / t=0$, then the double-dome-like surface $\Sigma(\Omega, \varphi)$ is not linearly locally connected, therefore not a quasisymmetric sphere, for any Jordan domain $\Omega$. The Lipschitz condition away from 0 , on the other hand, is added to tidy up the statements. For example, the surface $\Sigma\left(B^{2}(0,1), \varphi\right)$ associated to the gauge

$$
\varphi(t)= \begin{cases}1-\sqrt{1-t}, & t \in[0,1], \\ t, & t \in[1,+\infty),\end{cases}
$$

is not quasisymmetric to $\mathbb{S}^{2}$; however $\Sigma\left(B^{2}(0,2), \varphi\right)$ is.

In Section 3, we discuss properties of constant distance sets to Jordan curves. Starting from a 2-dimensional double-dome-like surface constructed over a planar quasidisk, we build quasispheres in all dimensions in Section 4 . The proof of Theorem 1.1 is given in Section 4.5. In Section 5, we establish a relation between the linear local connectedness of the surface $\Sigma(\Omega, \varphi)$ and the level quasicircle property of the domain $\Omega$. The proofs of Theorem 1.2 and Corollary 1.4 are completed in Section 6 .

\section{Preliminaries}

A homeomorphism $f: D \rightarrow D^{\prime}$ between two domains in $\mathbb{R}^{n}$ is called $K$-quasiconformal if it is orientation preserving, belongs to $W_{\text {loc }}^{1, n}(D)$, and satisfies the distortion inequality

$$
|D f(x)|^{n} \leq K J_{f}(x) \quad \text { a.e. } x \in D,
$$

where $D f$ is the formal differential matrix and $J_{f}$ is the Jacobian.

A Jordan curve $\gamma$ in $\mathbb{R}^{2}$ is called a $K$-quasicircle if it is the image of the unit circle $\mathbb{S}^{1}$ under a $K$-quasiconformal homeomorphism of $\mathbb{R}^{2}$. A geometric characterization due to Ahlfors [1] states that a Jordan curve $\gamma$ is a $K$-quasicircle if and only if it satisfies the 2-point condition:

$$
\text { there exists } C>1 \text { such that for all } x, y \in \gamma, \operatorname{diam} \gamma(x, y) \leq C|x-y| \text {, }
$$

where the distortion $K$ and the 2-point constant $C$ are quantitatively related. 
Images of $\mathbb{S}^{n}$ under quasiconformal homeomorphisms of $\mathbb{R}^{n+1}, n \geq 2$, are called quasispheres. The only known characterization of quasispheres is due to Gehring for $n=3$ in 1965 and to Väisälä for all $n \geq 3$ in 1984 .

Theorem 2.1 ([21, Theorem 5.9], [8, Theorem]). Let $n \geq 3$ and $\mathcal{S}$ be a topological $(n-1)$-sphere in $\mathbb{R}^{n}$. Suppose that components of $\mathbb{R}^{n} \backslash \mathcal{S}$ are $K$-quasiconformal to $\mathbb{B}^{n}$ and $\mathbb{R}^{n} \backslash \overline{\mathbb{B}^{n}}$ respectively. Then there is a $K^{\prime}$-quasiconformal homeomorphism of $\mathbb{R}^{n}$ that maps $\mathcal{S}$ onto $\mathbb{S}^{n-1}$, where constant $K^{\prime}>1$ depends only on $K$ and $n$.

Notice that this theorem is false when $n=2$.

An embedding $f$ of a metric space $\left(X, d_{X}\right)$ into a metric space $\left(Y, d_{Y}\right)$ is said to be $L$-bi-Lipschitz if there exists $L \geq 1$ such that for any $x, y \in X$,

$$
\frac{1}{L} d_{X}(x, y) \leq d_{Y}(f(x), f(y)) \leq L d_{X}(x, y) .
$$

A rectifiable Jordan curve $\gamma$ in $\mathbb{R}^{2}$ is called a c-chord-arc curve if there exists $c>1$ such that for any $x, y \in \gamma$, the length of the shorter arc $\gamma^{\prime}(x, y)$ in $\gamma \backslash\{x, y\}$ satisfies

$$
\ell\left(\gamma^{\prime}(x, y)\right) \leq c|x-y|
$$

It is straightforward to see that a rectifiable curve $\gamma$ is a $c$-chord-arc curve if and only if it satisfies

$$
\ell(\gamma(x, y)) \leq C|x-y|
$$

for all $x, y \in \gamma$ and some $C>1$; here constants $c$ and $C$ are quantitatively related.

Every $c$-chord-arc curve is, in fact, the image of $\mathbb{S}^{1}$ under an $L$-bi-Lipschitz homeomorphism of $\mathbb{R}^{2}$, where the constants $c$ and $L$ are quantitatively related; see [18, p. 23] and [13, Proposition 1.13].

An embedding $f$ of a metric space $\left(X, d_{X}\right)$ into a metric space $\left(Y, d_{Y}\right)$ is said to be $\eta$-quasisymmetric if there exists a homeomorphism $\eta:[0, \infty) \rightarrow[0, \infty)$ such that for all $x, a, b \in X$ and $t>0$ with $d_{X}(x, a) \leq t d_{X}(x, b)$,

$$
d_{Y}(f(x), f(a)) \leq \eta(t) d_{Y}(f(x), f(b)) .
$$

A metric $n$-sphere $\mathcal{S}$ that is quasisymmetrically homeomorphic to $\mathbb{S}^{n}$ is called a quasisymmetric sphere when $n \geq 2$ and a quasisymmetric circle when $n=1$.

Beurling and Ahlfors [2] showed that a planar Jordan curve is a quasisymmetric circle if and only if it is a quasicircle. Tukia and Väisälä [19] proved that a metric 1 -sphere is a quasisymmetric circle if and only if it is doubling and bounded turning.

The notion of linear local connectivity generalizes the 2-point condition on curves to general sets. A set $X \subset \mathbb{R}^{n}$ is $\lambda$-linearly locally connected (or $\lambda$-LLC) for $\lambda \geq 1$ if the following two conditions are satisfied.

(1) $\left(\lambda-\mathrm{LLC}_{1}\right)$ If $x \in X, r>0$ and $y_{1}, y_{2} \in B^{n}(x, r) \cap X$, then there exists a continuum $E \subset B^{n}(x, \lambda r) \cap X$ containing $y_{1}, y_{2}$.

(2) $\left(\lambda-\mathrm{LLC}_{2}\right)$ If $x \in X, r>0$ and $y_{1}, y_{2} \in X \backslash B^{n}(x, r)$, then there exists a continuum $E \subset X \backslash B^{n}(x, r / \lambda)$ containing $y_{1}, y_{2}$.

Linear local connectivity was first studied in the work of Gehring and Väisälä [11] and appeared, under the term strong local connectivity, in a paper of Gehring [8. In the latter, a set $X \subset \mathbb{R}^{n}$ is said to be strongly locally connected if it satisfies (1) and (2) for all $x \in \mathbb{R}^{n}$ instead of only for those $x \in X$. Walker 25] showed that any quasicircle is strongly locally connected.

Gehring and Väisälä [1] proved that if a domain $D \subset \mathbb{R}^{n}$ is quasiconformally equivalent to $\mathbb{B}^{n}$, then its complement $\mathbb{R}^{n} \backslash \bar{D}$ must be LLC. It is easy to check 
that the LLC property is preserved under quasisymmetry. As a consequence, every surface quasisymmetric to $\mathbb{S}^{n}$ or $\mathbb{R}^{n}$ satisfies the LLC property.

A metric space $X$ is said to be Ahlfors $Q$-regular if there is a constant $C>1$ such that the $Q$-dimensional Hausdorff measure $\mathcal{H}^{Q}$ of every open ball $B(a, r)$ in $X$ satisfies

$$
C^{-1} r^{Q} \leq \mathcal{H}^{Q}(B(a, r)) \leq C r^{Q}
$$

when $0<r \leq \operatorname{diam} X$.

Bonk and Kleiner found in [4] an intrinsic characterization of quasisymmetric 2 -spheres and then derived a readily applicable sufficient condition.

Theorem 2.2 (4, Theorem 1.1, Lemma 2.5]). Let $X$ be an Ahlfors 2-regular metric space homeomorphic to $\mathbb{S}^{2}$. Then $X$ is quasisymmetric to $\mathbb{S}^{2}$ if and only if $X$ is $L L C$.

For $x \in \mathbb{R}^{n}$ and $r>0$, define $B^{n}(x, r)=\left\{y \in \mathbb{R}^{n}:|x-y|<r\right\}$ and $S^{n-1}\left(x_{0}, r\right)=$ $\partial B^{n}\left(x_{0}, r\right)$; in particular, $\mathbb{B}^{n}=B^{n}(0,1)$ and $\mathbb{S}^{n-1}=\partial \mathbb{B}^{n}$. In addition, let

$$
\mathbb{R}_{+}^{n}=\left\{x=\left(x_{1}, \ldots, x_{n}\right) \in \mathbb{R}^{n}: x_{n} \leq 0\right\}
$$

be the upper half-space of $\mathbb{R}^{n}$ and $\mathbb{R}_{-}^{n}=\left\{x \in \mathbb{R}^{n}: x_{n} \leq 0\right\}$ be the lower half-space. For any $a=\left(a_{1}, \ldots, a_{n}\right) \in \mathbb{R}^{n}$, denote by

$$
\pi(a)=\left(a_{1}, \ldots, a_{n-1}, 0\right)
$$

the projection of $a$ on the hyperplane $\mathbb{R}^{n-1} \times\{0\}$. For $x, y \in \mathbb{R}^{n}$, denote by $[x, y]$ the line segment having endpoints $x, y$ and by $l_{x, y}$ the infinite line containing the points $x, y$.

In the following, we write $u \lesssim v$ (resp. $u \simeq v$ ) when the ratio $u / v$ is bounded above (resp. bounded above and below) by positive constants. These constants may vary but are described in each occurrence.

\section{LeVEL SETS TO A JoRdAN CURVE}

We discuss properties of constant distance sets to a Jordan curve that will be used in the sequel. The main reference is 24.

Let $\Omega$ be a planar Jordan domain with boundary $\Gamma$. We define for each $\epsilon>0$, the $\epsilon$-level set

$$
\gamma_{\epsilon}=\{x \in \Omega: \operatorname{dist}(x, \Gamma)=\epsilon\} .
$$

In general $\gamma_{\epsilon}$ need not be connected and if connected need not be a curve; see [24, Figure 1]. Properties of $\Omega$ ensuring the $\epsilon$-level sets to be Jordan curves, or uniform quasicircles, or uniform chord-arc curves for all sufficiently small $\epsilon$ have been studied in 24 .

We say $\Omega$ has the level Jordan curve property (or LJC property) if there exists $\epsilon_{0}>0$ such that the level set $\gamma_{\epsilon}$ is a Jordan curve for every $0 \leq \epsilon \leq \epsilon_{0}$. The Jordan domain $\Omega$ is said to have the level quasicircle property (or LQC property) if there exist $\epsilon_{0}>0$ and $K \geq 1$ such that the level set $\gamma_{\epsilon}$ is a $K$-quasicircle for every $0 \leq \epsilon \leq \epsilon_{0}$. Finally, the Jordan domain $\Omega$ is said to have the level chord-arc property (or LCA property) if there exist $\epsilon_{0}>0$ and $C \geq 1$ such that $\gamma_{\epsilon}$ is a $C$-chord-arc curve for every $0 \leq \epsilon \leq \epsilon_{0}$.

Sufficient conditions for a domain $\Omega$ to satisfy the LJC property or the LQC property can be given in terms of the chordal flatness of $\partial \Omega$. 
Proposition 3.1 ([24, Theorem 1.1, Theorem 1.2]). Let $\Omega$ be a Jordan domain.

(1) If there exists $r_{0}>0$ such that $\zeta_{\partial \Omega}(x, y)<1 / 2$ for all $x, y \in \partial \Omega$ with $|x-y|<r_{0}$, then $\Omega$ has the LJC property.

(2) If $\zeta_{\partial \Omega}<1 / 2$, then $\Omega$ has the $L Q C$ property.

In article [24, a Jordan curve $\Gamma$ is said to have one of the properties LJC, LQC, or LCA if both components of $\mathbb{R}^{2} \backslash \Gamma$ have that property.

A connection between the LQC property and the LCA property on $\Gamma$ is given in [24, Theorem 1.3]: A Jordan curve $\Gamma$ has the LCA property if and only if it is a chord-arc and has the LQC property. Since the proof of the LCA property for either component of $\mathbb{R}^{2} \backslash \Gamma$ requires only the LQC property of the same component, the following result can be concluded.

Proposition 3.2. A Jordan domain $\Omega$ in the plane satisfies the level chord-arc property if and only if it has the level quasicircle property and $\partial \Omega$ is a chord-arc curve.

We state two basic properties related to the distance function which are used repeatedly throughout the paper. For these and more, see [24, p. 216].

Assume that $\Omega$ is a planar Jordan domain and that points $x, y \in \Omega$ and $x^{\prime}, y^{\prime} \in$ $\partial \Omega$ satisfy $\left|x-x^{\prime}\right|=\operatorname{dist}(x, \partial \Omega)$ and $\left|y-y^{\prime}\right|=\operatorname{dist}(y, \partial \Omega)$. Then:

(non-crossing) the line segments $\left[x, x^{\prime}\right]\left[y, y^{\prime}\right]$ do not intersect except perhaps at their endpoints, unless $x, y, x^{\prime}, y^{\prime}$ are collinear;

(monotonicity) the distance function $\operatorname{dist}(\cdot, \partial \Omega)$ is strictly monotone on $\left[x, x^{\prime}\right]$. Moreover, if $z \in\left[x, x^{\prime}\right]$, then $\operatorname{dist}(z, \partial \Omega)=\left|z-x^{\prime}\right|$.

Lemma 3.3 ([24, Lemma 4.1]). Let $\Omega$ be a planar Jordan domain and $\Lambda \subset \partial \Omega$ be a closed subarc. Assume that for some $\epsilon>0$ the level set $\gamma_{\epsilon}$ is a Jordan curve. Then the set

$$
\left\{x \in \gamma_{\epsilon}: \operatorname{dist}(x, \Lambda)=\epsilon\right\},
$$

if non-empty, is a subarc of $\gamma_{\epsilon}$.

In general $\operatorname{dist}\left(z, \gamma_{\epsilon}\right)$ from points $z \in \Gamma=\partial \Omega$ to the level sets $\gamma_{\epsilon}$ need not have magnitude $\epsilon$, even when $\gamma_{\epsilon}$ are Jordan curves. The curve $\Gamma=\left\{(s, t):|t|=s^{2}, 0 \leq\right.$ $s<1\} \cup\{(1, t):-1 \leq t \leq 1\}$ in the Cartesian coordinates $(s, t)$ of the plane, is an example. This would not happen when $\Gamma$ is a quasicircle.

Lemma 3.4. Suppose that $\Omega$ is a Jordan domain whose boundary is a K-quasicircle and that $\gamma_{\epsilon}$ is a Jordan curve for some $\epsilon>0$. Then there exists $M \geq 1$ depending only on $K$ such that for any $x \in \partial \Omega, \epsilon \leq \operatorname{dist}\left(x, \gamma_{\epsilon}\right) \leq M \epsilon$.

Proof. Suppose that $x \in \partial \Omega$ is a point having $\operatorname{dist}\left(x, \gamma_{\epsilon}\right)>\epsilon$. By continuity, there exists a subarc $\Lambda$ of $\partial \Omega$ containing $x$ in its interior such that $\operatorname{dist}\left(p, \gamma_{\epsilon}\right)=$ $\operatorname{dist}\left(q, \gamma_{\epsilon}\right)=\epsilon$ at its endpoints $p$ and $q$ and that $\operatorname{dist}\left(y, \gamma_{\epsilon}\right)>\epsilon$ at each interior point $y$ of $\Lambda$. Take $\tilde{p}, \tilde{q} \in \gamma_{\epsilon}$ with $\operatorname{dist}\left(p, \gamma_{\epsilon}\right)=|p-\tilde{p}|$ and $\operatorname{dist}\left(q, \gamma_{\epsilon}\right)=|q-\tilde{q}|$, and let $\sigma$ be the component of $\gamma_{\epsilon} \backslash\{\tilde{p}, \tilde{q}\}$ which together with $\Lambda \cup[p, \tilde{p}] \cup[q, \tilde{q}]$ encloses a Jordan domain contained in $\Omega \backslash \Delta_{\epsilon}$. We further require that choices of $\tilde{p}, \tilde{q}$ are made in such a way that $\sigma$ is minimal.

We claim that $\tilde{p}=\tilde{q}$. Otherwise, take $z$ to be an interior point of $\sigma$ and take $z^{\prime}$ to be a point in $\Gamma$ with $\left|z-z^{\prime}\right|=\epsilon$. By the non-crossing property, $z^{\prime}$ must be in the interior of $\Lambda$; this contradicts the definition of $\Lambda$. Therefore $\tilde{p}$ and $\tilde{q}$ must be the same, which implies that $|p-q| \leq 2 \epsilon$. 
Since $\partial \Omega$ is a $K$-quasicircle, there exists $C>1$ depending only on $K$ so that

$$
\operatorname{dist}\left(x, \gamma_{\epsilon}\right) \leq|x-p|+|p-\tilde{p}| \leq \operatorname{diam} \Lambda+\epsilon \leq C|p-q|+\epsilon \leq(2 C+1) \epsilon .
$$

For a Jordan domain $\Omega$ and a number $\epsilon>0$, consider the open sets

$$
\Delta_{\epsilon}=\{x \in \Omega: \operatorname{dist}(x, \Omega)>\epsilon\} .
$$

In general $\Delta_{\epsilon}$ need not be connected, and $\overline{\Delta_{\epsilon}}$ and $\Delta_{\epsilon} \cup \gamma_{\epsilon}$ may not be the same. Nevertheless we have the following.

Lemma 3.5 ([24, Lemma 4.5]). Let $\Omega$ be a Jordan domain and $\epsilon>0$. Then, every connected component of $\Delta_{\epsilon}$ is a Jordan domain.

Lemma 3.6 ([24, Remark 4.13]). Suppose that $\Omega$ is a Jordan domain and that for some $\epsilon>0, \Delta_{\epsilon} \neq \emptyset, \gamma_{\epsilon} \cup \Delta_{\epsilon}$ is connected, and $\bar{\Delta}_{\epsilon} \subsetneq \gamma_{\epsilon} \cup \Delta_{\epsilon}$. Then, there exist a component $D$ of $\Delta_{\epsilon}$ and collinear points $x_{0} \in \partial D$ and $x_{1}, x_{2} \in \Gamma$ such that

$$
\left|x_{0}-x_{1}\right|=\left|x_{0}-x_{2}\right|=\epsilon .
$$

Lemma 3.7 ([24, Remark 4.15]). Suppose that $\Omega$ is a Jordan domain and that for some $\epsilon>0, \Delta_{\epsilon} \neq \emptyset$ and $\gamma_{\epsilon} \cup \Delta_{\epsilon}$ is not connected. Then, there exist $\delta \in(0, \epsilon), a$ component $D$ of $\Delta_{\delta}$, and collinear points $x_{0} \in \partial D$ and $x_{1}, x_{2} \in \Gamma$ such that

$$
\left|x_{0}-x_{1}\right|=\left|x_{0}-x_{2}\right|=\delta \text {. }
$$

\section{QUASISPHERES OVER QUASIDISKS}

We prove Theorem 1.1 in this section. For the proof, we need some well-known inequalities from the classical function theory.

4.1. Koebe estimates. The Koebe $1 / 4$ Theorem states that if $f$ is a conformal map from the unit disk $\mathbb{B}^{2}$ onto a simply connected domain $\Omega$, then

$$
\left|f^{\prime \prime}(0) / f^{\prime}(0)\right| \leq 2
$$

and

$$
\operatorname{dist}(f(0), \partial \Omega) \geq\left|f^{\prime}(0)\right| / 4 \text {. }
$$

It follows by scaling that for all $x \in \mathbb{B}^{2}$,

$$
\frac{\operatorname{dist}(f(x), \partial \Omega)}{1-|x|^{2}} \leq\left|f^{\prime}(x)\right| \leq \frac{4 \operatorname{dist}(f(x), \partial \Omega)}{1-|x|^{2}} .
$$

In fact, there exists an absolute constant $A>1$ so that for all $x_{0} \in \mathbb{B}^{2}$ and $x, x_{1}, x_{2} \in B^{2}\left(x_{0},\left(1-\left|x_{0}\right|\right) / 2\right) \subset \mathbb{B}^{2}$,

$$
\frac{1}{A} \frac{\left|f\left(x_{1}\right)-f\left(x_{2}\right)\right|}{\left|x_{1}-x_{2}\right|} \leq\left|f^{\prime}(x)\right| \leq A \frac{\left|f\left(x_{1}\right)-f\left(x_{2}\right)\right|}{\left|x_{1}-x_{2}\right|}
$$

and

$$
\operatorname{dist}\left(f\left(x_{0}\right), \partial \Omega\right) / A \leq \operatorname{diam} f\left(B^{2}\left(x_{0},\left(1-\left|x_{0}\right|\right) / 2\right)\right) \leq A \operatorname{dist}\left(f\left(x_{0}\right), \partial \Omega\right) .
$$

See for example the books [6] and [17]. 
Set $D_{x}=\frac{\operatorname{dist}(f(x), \partial \Omega)}{1-|x|}$. Then it follows from (4.1), (4.2) and the Lipschitz continuity of the distance function $\operatorname{dist}(\cdot, \partial \Omega)$ that for all $x_{0} \in \mathbb{B}^{2}$ and $x_{1}, x_{2} \in$ $B^{2}\left(x_{0},\left(1-\left|x_{0}\right|\right) / 2\right) \subset \mathbb{B}^{2}$,

$$
\begin{aligned}
\mid D_{x_{1}} & -D_{x_{2}}|=| \frac{\operatorname{dist}\left(f\left(x_{1}\right), \partial \Omega\right)}{1-\left|x_{1}\right|}-\frac{\operatorname{dist}\left(f\left(x_{2}\right), \partial \Omega\right)}{1-\left|x_{2}\right|} \mid \\
& \leq \frac{\left(1-\left|x_{2}\right|\right)\left|\operatorname{dist}\left(f\left(x_{1}\right), \partial \Omega\right)-\operatorname{dist}\left(f\left(x_{2}\right), \partial \Omega\right)\right|+\left|x_{1}-x_{2}\right| \operatorname{dist}\left(f\left(x_{2}\right), \partial \Omega\right)}{\left(1-\left|x_{1}\right|\right)\left(1-\left|x_{2}\right|\right)} \\
& \leq \frac{\left|f\left(x_{1}\right)-f\left(x_{2}\right)\right|}{1-\left|x_{1}\right|}+\frac{\left|x_{1}-x_{2}\right|}{1-\left|x_{1}\right|} \frac{\operatorname{dist}\left(f\left(x_{2}\right), \partial \Omega\right)}{1-\left|x_{2}\right|} \\
& \leq 8 A^{2} \frac{\left|f^{\prime}\left(x_{0}\right)\right|\left|x_{1}-x_{2}\right|}{1-\left|x_{0}\right|} .
\end{aligned}
$$

4.2. A class of 3-dimensional quasiballs. We show that for any simply connected domain $\Omega$ in $\mathbb{R}^{2}$, the domain enclosed by the double-dome-like surface $\Sigma(\Omega, t)$ is quasiconformally equivalent to the unit ball $\mathbb{B}^{3}$.

Theorem 4.1. Let $\Omega$ be a simply connected domain in $\mathbb{R}^{2}$. Then the double cone with base $\Omega$,

$$
\mathcal{K}(\Omega, t)=\{(x, z): x \in \Omega,|z|<\operatorname{dist}(x, \partial \Omega)\},
$$

is $K_{0}$-quasiconformal homeomorphic to the unit ball $\mathbb{B}^{3}$, for some absolute constant $K_{0}>1$.

Proof. Define first a double circular cone $\mathcal{C}$ with base $\mathbb{B}^{2}$,

$$
\mathcal{C}=\left\{(x, z) \in \mathbb{B}^{2} \times \mathbb{R}:|z|<1-|x|\right\} .
$$

Fix a conformal map $f$ from $\mathbb{B}^{2}$ onto $\Omega$ and define a homeomorphism $F: \mathcal{C} \rightarrow \mathcal{K}(\Omega, t)$ between cones, associated to $\Omega$ and $f$, by

$$
F(x, z)=\left(f(x), \frac{\operatorname{dist}(f(x), \partial \Omega)}{1-|x|} z\right) .
$$

We claim that $F$ is $K_{1}$-quasiconformal for some absolute constant $K_{1}>1$. The theorem follows from the claim and the fact that the cone $\mathcal{C}$ is quasiconformally equivalent to $\mathbb{B}^{3}$.

To this end, take $x_{0} \in \mathbb{B}^{2}$ and $\left(x_{1}, z_{1}\right),\left(x_{2}, z_{2}\right)$ in $\mathcal{C}$ with $x_{1}, x_{2} \in$ $B^{2}\left(x_{0},\left(1-\left|x_{0}\right|\right) / 2\right) \subset \mathbb{B}^{2}$ and $-1<z_{1}, z_{2}<1$. Inequalities (4.1), (4.2) and (4.3) yield

$$
\begin{aligned}
\left|F\left(x_{1}, z_{1}\right)-F\left(x_{2}, z_{2}\right)\right| & \leq\left|f\left(x_{1}\right)-f\left(x_{2}\right)\right|+\left|D_{x_{1}} z_{1}-D_{x_{2}} z_{2}\right| \\
& \leq A\left|f^{\prime}\left(x_{0}\right)\right|\left|x_{1}-x_{2}\right|+\left|D_{x_{1}}-D_{x_{2}}\right|\left|z_{2}\right|+D_{x_{1}}\left|z_{1}-z_{2}\right| \\
& \leq A\left|f^{\prime}\left(x_{0}\right)\right|\left|x_{1}-x_{2}\right|+\frac{3}{2}\left|D_{x_{1}}-D_{x_{2}}\right|\left(1-\left|x_{0}\right|\right) \\
& \quad+2 A^{2}\left|f^{\prime}\left(x_{0}\right)\right|\left|z_{1}-z_{2}\right| \\
& \leq 15 A^{2}\left|f^{\prime}\left(x_{0}\right)\right|\left|\left(x_{1}, z_{1}\right)-\left(x_{2}, z_{2}\right)\right| .
\end{aligned}
$$


For a lower estimate, note that when $\left|z_{1}-z_{2}\right| \leq 60 A^{4}\left|x_{1}-x_{2}\right|$,

$$
\begin{aligned}
\left|F\left(x_{1}, z_{1}\right)-F\left(x_{2}, z_{2}\right)\right| & \geq\left|f\left(x_{1}\right)-f\left(x_{2}\right)\right| \\
& \geq \frac{1}{A}\left|f^{\prime}\left(x_{0}\right)\right|\left|x_{1}-x_{2}\right| \\
& \geq \frac{1}{61 A^{5}}\left|f^{\prime}\left(x_{0}\right)\right|\left|\left(x_{1}, z_{1}\right)-\left(x_{2}, z_{2}\right)\right| .
\end{aligned}
$$

On the other hand, if $\left|z_{1}-z_{2}\right|>60 A^{4}\left|x_{1}-x_{2}\right|$, then

$$
\begin{aligned}
\left|F\left(x_{1}, z_{1}\right)-F\left(x_{2}, z_{2}\right)\right| & \geq\left|D_{x_{1}} z_{1}-D_{x_{2}} z_{2}\right| \\
& \geq D_{x_{2}}\left|z_{1}-z_{2}\right|-\left|\left(D_{x_{1}}-D_{x_{2}}\right) z_{1}\right| \\
& \geq \frac{1}{4}\left|f^{\prime}\left(x_{2}\right)\right|\left|z_{1}-z_{2}\right|-8 A^{2} \frac{\left|f^{\prime}\left(x_{0}\right)\right|\left|x_{1}-x_{2}\right|}{1-\left|x_{0}\right|}\left|z_{1}\right| \\
& \geq \frac{1}{4 A^{2}}\left|f^{\prime}\left(x_{0}\right)\right|\left|z_{1}-z_{2}\right|-12 A^{2}\left|f^{\prime}\left(x_{0}\right)\right|\left|x_{1}-x_{2}\right| \\
& \geq \frac{1}{30 A^{2}}\left|f^{\prime}\left(x_{0}\right)\right|\left|\left(x_{1}, z_{1}\right)-\left(x_{2}, z_{2}\right)\right| .
\end{aligned}
$$

From these estimates it follows that the restrictions of $\frac{F}{\left|f^{\prime}\left(x_{0}\right)\right|}$ on $\mathcal{C} \cap$ $\left(B^{2}\left(x_{0},\left(1-\left|x_{0}\right|\right) / 2\right) \times \mathbb{R}\right)$ are uniformly bi-Lipschitz for all $x_{0} \in \mathbb{B}^{2}$. Hence $F$ is $K_{1}$ quasiconformal for some absolute $K_{1}>1$.

4.3. Higher dimensional quasiballs. The procedure of constructing quasiballs in $\mathbb{R}^{3}$ extends to all dimensions.

Theorem 4.2. Let $\Omega$ be a simply connected domain in $\mathbb{R}^{2}$, set $\mathcal{K}^{3}(\Omega, t)=\mathcal{K}(\Omega, t)$, the cone defined in (4.4), and write

$$
\mathcal{K}^{n}(\Omega, t)=\left\{(x, z): x \in \mathcal{K}^{n-1}(\Omega, t),|z|<\operatorname{dist}\left(x, \partial \mathcal{K}^{n-1}(\Omega, t)\right)\right\}
$$

for $n \geq 4$. Then for any $n \geq 3, \mathcal{K}^{n}(\Omega, t)$ is quasiconformally homeomorphic to the unit ball $\mathbb{B}^{n}$.

We give an outline of the proof.

Set $\mathcal{C}^{3}=\mathcal{C}$ and

$$
\mathcal{C}^{n}=\left\{(x, z): x \in \mathcal{C}^{n-1},|z|<\operatorname{dist}\left(x, \partial \mathcal{C}^{n-1}\right)\right\}
$$

for $n \geq 4$. The quasiconformality of the map $F: \mathcal{C}^{3} \rightarrow \mathcal{K}^{3}(\Omega, t)$ was deduced from the Lipschitz property of a distance function and the Koebe estimates (4.1) and (4.2). These estimates are essentially equivalent to the following:

(a) for any $x_{0} \in \mathbb{B}^{2}$, the restriction $\left.f\right|_{\mathbb{B}^{2}\left(x_{0},\left(1-\left|x_{0}\right|\right) / 2\right)}$ is $C\left(x_{0}\right)$-bi-Lipschitz,

(b) the bi-Lipschitz constant $C\left(x_{0}\right)$ is comparable to $\frac{\operatorname{dist}\left(f\left(x_{0}\right), \partial \Omega\right)}{\operatorname{dist}\left(x_{0}, \partial \mathbb{B}^{2}\right)}$ with ratios bounded above and below by constants independent of $x_{0}$.

The extended map $F: \mathcal{C}^{3} \rightarrow \mathcal{K}^{3}(\Omega, t)$ defined in (4.6) inherits properties (a) and (b) from that of $f$. Indeed, the proof of Theorem 4.1 implies that there exists an absolute constant $A_{1}>1$ such that for any $\left(x_{0}, z_{0}\right) \in \mathcal{C}^{3}$ and $(x, z),\left(x_{1}, z_{1}\right),\left(x_{2}, z_{2}\right) \in$ $B^{3}\left(\left(x_{0}, z_{0}\right), \frac{1}{2} \operatorname{dist}\left(\left(x_{0}, z_{0}\right), \partial \mathcal{C}^{3}\right)\right) \subset \mathcal{C}^{3}$,

$$
\frac{1}{A_{1}} \frac{\left|F\left(x_{1}, z_{1}\right)-F\left(x_{2}, z_{2}\right)\right|}{\left|\left(x_{1}, z_{1}\right)-\left(x_{2}, z_{2}\right)\right|} \leq\left|f^{\prime}(x)\right| \leq A_{1} \frac{\left|F\left(x_{1}, z_{1}\right)-F\left(x_{2}, z_{2}\right)\right|}{\left|\left(x_{1}, z_{1}\right)-\left(x_{2}, z_{2}\right)\right|} .
$$


Since quasiconformal mappings map Whitney-type cubes to Whitney-type sets ( 7 . Theorem 11], [12, p. 93]), we can deduce from (4.7) that

$$
\frac{1}{A_{2}} \frac{\operatorname{dist}\left(F\left(x_{0}, z_{0}\right), \partial \mathcal{K}^{3}(\Omega, t)\right)}{\operatorname{dist}\left(\left(x_{0}, z_{0}\right), \partial \mathcal{C}^{3}\right)} \leq\left|f^{\prime}\left(x_{0}\right)\right| \leq A_{2} \frac{\operatorname{dist}\left(F\left(x_{0}, z_{0}\right), \partial \mathcal{K}^{3}(\Omega, t)\right)}{\operatorname{dist}\left(\left(x_{0}, z_{0}\right), \partial \mathcal{C}^{3}\right)}
$$

for some $A_{2}>1$ depending only on the dilatation $K_{0}$ in Theorem 4.1

Estimates (4.7) and (4.8) and the Lipschitz property of a distance function allow the induction steps to continue. As a consequence, the map

$$
G:\left(x, z, z^{\prime}\right) \rightarrow\left(F(x, z), \frac{\operatorname{dist}\left(F(x, z), \partial \mathcal{K}^{3}(\Omega, t)\right)}{\operatorname{dist}\left((x, z), \partial \mathcal{C}^{3}\right)} z^{\prime}\right)
$$

for $(x, z) \in \mathcal{C}^{3}$ and $\left|z^{\prime}\right|<\operatorname{dist}\left((x, z), \partial \mathcal{C}^{3}\right)$ is quasiconformal from $\mathcal{C}^{4}$ onto $\mathcal{K}^{4}(\Omega, t)$, and it satisfies the local bi-Lipschitz properties (a) and (b) needed for the next step. In particular, the restrictions of $\frac{G}{\left|f^{\prime}\left(x_{0}\right)\right|}$ on

$$
B^{4}\left(\left(x_{0}, z_{0}, z_{0}^{\prime}\right), \frac{1}{2} \operatorname{dist}\left(\left(x_{0}, z_{0}, z_{0}^{\prime}\right), \partial \mathcal{C}^{4}\right)\right)
$$

are uniformly bi-Lipschitz for all $\left(x_{0}, z_{0}, z_{0}^{\prime}\right) \in \mathcal{C}^{4}$. Since $\mathcal{C}^{n}$ is bi-Lipschitz equivalent to $\mathbb{B}^{n}, \mathcal{K}^{n}(\Omega, t)$ is quasiconformal to $\mathbb{B}^{n}$ for any $n \geq 3$.

4.4. Slit domains. A domain $D \subset \mathbb{R}^{n}$ is called a slit domain if $\mathbb{R}^{n} \backslash \bar{D} \subset \mathbb{R}^{n-1} \times$ $\{0\}$. A theorem of Gehring on quasiconformal maps on slit domains in $\mathbb{R}^{3}$ is stated as follows.

Theorem 4.3 ([9, Theorem 5]). Suppose that $\Omega$ is a planar Jordan domain. Then the slit domain $\mathbb{R}^{3} \backslash \bar{\Omega}$ is quasiconformally homeomorphic to $\mathbb{R}^{3} \backslash \overline{\mathbb{B}^{3}}$ if and only if $\Omega$ is a quasidisc.

We state a simple observation on slit domains that is needed later.

Lemma 4.4. Let $D$ be a bounded domain in $\mathbb{R}^{n}$ and $h_{1}, h_{2}: \mathbb{R}^{n} \rightarrow \mathbb{R}$ be L-Lipschitz functions satisfying $h_{2} \leq h_{1}$ in $D$ and $h_{1}=h_{2}=0$ in $\mathbb{R}^{n} \backslash D$. Then the domain

$$
\mathbb{R}^{n+1} \backslash\left\{(x, z): x \in D, h_{2}(x) \leq z \leq h_{1}(x)\right\}
$$

is locally $(L+2)$-bi-Lipschitz to, therefore $K$-quasiconformal to, the slit domain $\mathbb{R}^{n+1} \backslash \bar{D}$, for some $K \geq 1$ depending only on $L$.

Proof. Define $H: \mathbb{R}^{n+1} \backslash \bar{D} \rightarrow \mathbb{R}^{n+1} \backslash\left\{(x, z): x \in \bar{D}, h_{2}(x) \leq z \leq h_{1}(x)\right\}$ with

$$
H(x, z)= \begin{cases}\left(x, z+h_{1}(x)\right) & \text { if } x \in \bar{D} \text { and } z>0 \\ \left(x, z+h_{2}(x)\right) & \text { if } x \in \bar{D} \text { and } z<0 \\ (x, z) & \text { if } x \notin \bar{D}\end{cases}
$$

It is straightforward to check that $H$ is $(L+2)$-bi-Lipschitz in each of the two halfspaces $\left\{(x, z): x \in \mathbb{R}^{n}, z>0\right\}$ and $\left\{(x, z): x \in \mathbb{R}^{n}, z<0\right\}$. Since $H$ is homeomorphic on $\mathbb{R}^{n+1} \backslash \bar{D}$, it is locally $(L+2)$-bi-Lipschitz and therefore $K$-quasiconformal for some $K$ depending only on $L$. 
4.5. Proof of Theorem 1.1. For the sufficiency in Theorem 1.1, we apply Theorem 2.1 of Gehring and Väisälä. Note first from Theorem 4.1 that the bounded component of $\mathbb{R}^{3} \backslash \Sigma(\Omega, t)$ is quasiconformal to $\mathbb{B}^{3}$. On the other hand, by Lemma 4.4, the unbounded component of $\mathbb{R}^{3} \backslash \Sigma(\Omega, t)$ is quasiconformally homeomorphic to the slit domain $\mathbb{R}^{3} \backslash \bar{\Omega}$. Since $\Omega$ is a planar quasidisk, $\mathbb{R}^{3} \backslash \bar{\Omega}$ is quasiconformal to the exterior $\mathbb{R}^{3} \backslash \overline{\mathbb{B}^{3}}$ of the unit ball by Theorem 4.3 . Therefore, $\Sigma(\Omega, t)$ is a quasisphere by Theorem 2.1.

It remains to prove the necessity.

Suppose that the double-dome-like surface $\Sigma(\Omega, t)$ is a $K$-quasisphere, hence a quasisymmetric sphere. Therefore $\Sigma(\Omega, t)$ is $\lambda$ - LLC for some $\lambda>1$ depending only on $K$. We claim that $\partial \Omega$ satisfies the 2-point condition (2.1) with $C=32 \lambda^{2}$.

Suppose the claim is false. Then there exist points $x_{1}, x_{2}, x_{3}, x_{4} \in \partial \Omega$ in cyclic order such that

$$
\left|x_{2}-x_{1}\right|,\left|x_{4}-x_{1}\right|>32 \lambda^{2}\left|x_{3}-x_{1}\right|
$$

Define

$$
d=\inf \left\{\operatorname{diam} \sigma: \sigma \subset \bar{\Omega} \text { is a continuum that contains } x_{1} \text { and } x_{3}\right\} .
$$

Since $x_{1}$ and $x_{3}$ are in the set $\Sigma(\Omega, t) \cap B^{3}\left(x_{1}, 2\left|x_{1}-x_{3}\right|\right)$, they are, by $\lambda-\mathrm{LLC}_{1}$, contained in a continuum $E$ in $\Sigma(\Omega, t) \cap B^{3}\left(x_{1}, 2 \lambda\left|x_{1}-x_{3}\right|\right)$, and hence in the projection $\pi(E)$. Thus

$$
d \leq \operatorname{diam} \pi(E) \leq \operatorname{diam} E \leq 4 \lambda\left|x_{1}-x_{3}\right| .
$$

Next fix a continuum $\sigma$ in $\Omega$ that contains $x_{1}$ and $x_{3}$ and has diam $\sigma \leq 4 \lambda\left|x_{1}-x_{3}\right|$. Since points $x_{2}$ and $x_{4}$ are in the set $\Sigma(\Omega, t) \backslash B^{3}\left(x_{1}, 16 \lambda^{2}\left|x_{1}-x_{3}\right|\right)$, they are, by the $\lambda-\mathrm{LLC}_{2}$ property, contained in a continuum $F$ in $\Sigma(\Omega, t) \backslash B^{3}\left(x_{1}, 16 \lambda\left|x_{1}-x_{3}\right|\right)$, and hence in the projection $\pi(F)$. Since $\pi(F)$ is a continuum in $\bar{\Omega}$, it intersects $\sigma$. Take a point $w \in F$ with $\pi(w) \in \sigma \cap \pi(F)$. Hence,

$$
\begin{aligned}
16 \lambda\left|x_{1}-x_{3}\right|<\left|w-x_{1}\right| & \leq|w-\pi(w)|+\left|\pi(w)-x_{1}\right| \\
& =\operatorname{dist}(\pi(w), \partial \Omega)+\left|\pi(w)-x_{1}\right| \\
& \leq 2\left|\pi(w)-x_{1}\right| \leq 8 \lambda\left|x_{1}-x_{3}\right|,
\end{aligned}
$$

which is a contradiction. Therefore $\partial \Omega$ satisfies the 2-point condition with $C=$ $32 \lambda^{2}$; hence it is a quasicircle.

This completes the proof of Theorem 1.1

The proof in fact shows the following.

Remark 4.5. If the double-dome-like surface $\Sigma(\Omega, t)$ is LLC, then $\partial \Omega$ is a quasicircle.

4.6. Higher dimensional quasispheres. The method of constructing quasispheres extends to all dimensions.

Theorem 4.6. Suppose that $\Omega$ is a quasidisk in $\mathbb{R}^{2}$. Then $\partial\left(\mathcal{K}^{n}(\Omega, t)\right)$ is a quasisphere in $\mathbb{R}^{n}$ for every $n \geq 3$.

Proof. Since $\mathcal{K}^{n}(\Omega, t)$ is quasiconformal to $\mathbb{B}^{n}$ by Theorem 4.2 it suffices to check that $\mathbb{R}^{n} \backslash \overline{\mathcal{K}^{n}(\Omega, t)}$ is quasiconformal to $\mathbb{R}^{n} \backslash \overline{\mathbb{B}^{n}}$ in view of Theorem 2.1

Fix, by Theorem 1.1, a quasiconformal $g: \mathbb{R}^{3} \rightarrow \mathbb{R}^{3}$ that maps $\mathcal{C}^{3}$ onto $\mathcal{K}^{3}(\Omega, t)$. Extend $g$, by a quasiconformal extension theorem of Tukia and Väisälä [20, Theorem $3.12]$, to a quasiconformal homeomorphism $G: \mathbb{R}^{4} \rightarrow \mathbb{R}^{4}$ with $\left.G\right|_{\mathbb{R}^{3} \times\{0\}}=g$; hence $\mathbb{R}^{4} \backslash \overline{\mathcal{C}^{3}}$ is quasiconformal to $\mathbb{R}^{4} \backslash \overline{\mathcal{K}^{3}(\Omega, t)}$. Furthermore, by Lemma $4.4, \mathbb{R}^{4} \backslash \overline{\mathcal{K}^{3}(\Omega, t)}$ 
is quasiconformal to $\mathbb{R}^{4} \backslash \overline{\mathcal{K}^{4}(\Omega, t)}$, and $\mathbb{R}^{4} \backslash \overline{\mathcal{C}^{3}}$ is quasiconformal to $\mathbb{R}^{4} \backslash \overline{\mathcal{C}^{4}}$, hence quasiconformal to $\mathbb{R}^{4} \backslash \overline{\mathbb{B}^{4}}$. Therefore $\mathbb{R}^{4} \backslash \overline{\mathcal{K}^{4}(\Omega, t)}$ is quasiconformal to $\mathbb{R}^{4} \backslash \overline{\mathbb{B}^{4}}$. This procedure can be continued inductively to all dimensions. We conclude that $\mathbb{R}^{n} \backslash \overline{\mathcal{K}^{n}(\Omega, t)}$ is quasiconformal to $\mathbb{R}^{n} \backslash \overline{\mathbb{B}^{n}}$ for any $n \geq 3$.

Remark 4.7. Theorem 4.6 remains true if we replace $\varphi(t)=t$ with gauges $\varphi \in \mathcal{F}$ that are bi-Lipschitz on $[0, \infty)$.

Indeed, suppose that $\Omega$ is a $K$-quasidisk and $\varphi_{1}, \varphi_{2}, \ldots$ are $L_{i}$-bi-Lipschitz gauges in $\mathcal{F}$. Set

$$
\mathcal{K}^{3}=\left\{(x, z): x \in \Omega,|z|<\varphi_{1}(\operatorname{dist}(x, \partial \Omega))\right\}
$$

and

$$
\mathcal{K}^{n+2}=\left\{(x, z): x \in \mathcal{K}^{n+1},|z|<\varphi_{n}\left(\operatorname{dist}\left(x, \partial \mathcal{K}^{n+1}\right)\right)\right\}
$$

for $n \geq 2$. Then, for any $n \geq 1$, the surface $\partial \mathcal{K}^{n+2}$ is a $K_{n}$-quasisphere in $\mathbb{R}^{n+2}$, with $K_{n}$ depending only on $n, K, L_{1}, \ldots, L_{n}$.

\section{LINEAR LOCAL CONNECTIVITY AND THE LEVEL QUASICIRCLE PROPERTY}

In this section we establish a relation between the LLC property of $\Sigma(\Omega, \varphi)$ and the LQC property on $\Omega$.

Proposition 5.1. Let $\Omega$ be a Jordan domain. Then the surface $\Sigma(\Omega, \varphi)$ is LLC for all $\varphi \in \mathcal{F}$ if and only if $\Omega$ has the $L Q C$ property.

We need a stronger form of $\mathrm{LLC}_{2}$ for planar quasicircles for Lemma 5.3, the straightforward proof is left to the reader.

Remark 5.2. Let $\Gamma \subset \mathbb{R}^{2}$ be a $K$-quasicircle. Then there exists $\lambda=\lambda(K)>1$ such that for any $x \in \mathbb{R}^{2}$, any $r>0$, and any two points $y_{1}, y_{2} \in \Gamma \backslash \bar{B}^{2}(x, r)$, there exists a subarc in $\Gamma \backslash B^{2}(x, r / \lambda)$ that contains $y_{1}$ and $y_{2}$.

From here onward, given $\Omega$ and $\varphi$ we set

$$
\Sigma(\Omega, \varphi)^{+}=\Sigma(\Omega, \varphi) \cap \mathbb{R}_{+}^{3} \text { and } \Sigma(\Omega, \varphi)^{-}=\Sigma(\Omega, \varphi) \cap \mathbb{R}_{-}^{3},
$$

and for a given subset $S$ of $\bar{\Omega}$, let

$$
S^{+}=\{(x, \varphi(\operatorname{dist}(x, \partial \Omega))): x \in S\}
$$

and

$$
S^{-}=\{(x,-\varphi(\operatorname{dist}(x, \partial \Omega))): x \in S\},
$$

be the lifts of $S$ to $\Sigma(\Omega, \varphi)^{+}$and $\Sigma(\Omega, \varphi)^{-}$, respectively. For instance, $\Delta_{\epsilon}^{-}$is the part of surface in $\Sigma(\Omega, \varphi)^{-}$whose projection on $\mathbb{R}^{2} \times\{0\}$ is $\Delta_{\epsilon}$, and $w^{+}=$ $(w, \varphi(\operatorname{dist}(w, \partial \Omega)))$ when $w$ is a point in $\Omega$.

\subsection{Sufficient conditions for $\Sigma(\Omega, \varphi)$ to be LLC.}

Lemma 5.3. Let $\Omega$ be a Jordan domain that has the level quasicircle property $(L Q C)$ and $\varphi$ be a homeomorphism in $\mathcal{F}$. In particular, there exist $K, L, M>1$ and $\epsilon_{0}>0$ so that $\gamma_{\epsilon}$ is a K-quasicircle for every $\epsilon \in\left[0, \epsilon_{0}\right]$ and so that $\varphi$ is L-Lipschitz in $\left[\epsilon_{0} / 3, \infty\right)$ and satisfies

$$
\varphi(t)>M t \text { for all } t \in[0, \operatorname{diam} \Gamma] .
$$

Then, $\Sigma(\Omega, \varphi)$ is $\lambda-L L C$ for some $\lambda>1$ depending only on $K, L, M$, $\epsilon_{0}$ and $\operatorname{diam} \Omega$. 
Proof. Since all level curves $\left\{\gamma_{\epsilon}\right\}_{0 \leq \epsilon<\epsilon_{0}}$ are $K$-quasicircles, they satisfy the 2-point condition (2.1) with a common $C>1$.

Part I. The $\mathrm{LLC}_{1}$ property.

We claim that there exists $\lambda>1$ such that for any $y_{1}$ and $y_{2}$ in $\Sigma(\Omega, \varphi)$, there exists a curve $\sigma$ in $\Sigma(\Omega, \varphi)$ joining $y_{1}, y_{2}$ and having diam $\sigma \leq \lambda\left|y_{1}-y_{2}\right|$. This statement implies that $\Sigma(\Omega, \varphi)$ is $(1+2 \lambda)-\mathrm{LLC}_{1}$. For the proof of the claim we consider three cases.

Case 1 . Suppose that both $y_{1}$ and $y_{2}$ are in $\overline{\Delta_{\epsilon_{0}}^{+}}$or both are in $\overline{\Delta_{\epsilon_{0}}^{-}}$. Since $\Delta_{\epsilon_{0}}$ is a $K$-quasidisk, $\overline{\Delta_{\epsilon_{0}}}$ contains an $\operatorname{arc} \tau$ joining $\pi\left(y_{1}\right)$ to $\pi\left(y_{2}\right)$ whose diameter is at most $C\left|\pi\left(y_{1}\right)-\pi\left(y_{2}\right)\right|$. Because $\varphi$ is $L$-Lipschitz on $\left[\epsilon_{0} / 3, \infty\right), \tau$ lifts to a curve $\sigma=\tau^{+}$ on $\Sigma(\Omega, \varphi)$ which connects $y_{1}$ to $y_{2}$ and has diam $\sigma \leq(C+L)\left|\pi\left(y_{1}\right)-\pi\left(y_{2}\right)\right|$.

Case 2. Suppose that both $y_{1}$ and $y_{2}$ are in the same half-space and at least one of them is not in $\overline{\Delta_{\epsilon_{0}}^{+}} \cup \overline{\Delta_{\epsilon_{0}}^{-}}$. Assume, for instance, that $y_{1}, y_{2} \in \mathbb{R}_{+}^{3}, y_{1} \in \Sigma(\Omega, \varphi)^{+} \backslash \Delta_{\epsilon_{0}}^{+}$ and $\pi\left(y_{1}\right) \in \gamma_{\epsilon_{1}}$ and $\pi\left(y_{2}\right) \in \gamma_{\epsilon_{2}}$ with $\epsilon_{1} \leq \epsilon_{2}$. Other subcases can be treated analogously.

Take $w$ in $\Sigma(\Omega, \varphi)^{+}$such that $\pi(w)$ is a point on $\gamma_{\epsilon_{1}}$ that is nearest to $\pi\left(y_{2}\right)$; thus $\left|\pi\left(y_{2}\right)-\pi(w)\right|=\epsilon_{2}-\epsilon_{1}$.

Since $\varphi$ is increasing in $\left[\epsilon_{1}, \epsilon_{2}\right]$ we have that

$$
\begin{aligned}
\left|y_{2}-w\right| & \leq\left|\pi\left(y_{2}\right)-\pi(w)\right|+\varphi\left(\epsilon_{2}\right)-\varphi\left(\epsilon_{1}\right) \\
& \leq\left|\pi\left(y_{2}\right)-\pi\left(y_{1}\right)\right|+\varphi\left(\epsilon_{2}\right)-\varphi\left(\epsilon_{1}\right) \\
& \leq 2\left|y_{1}-y_{2}\right|
\end{aligned}
$$

and

$$
\begin{aligned}
\left|y_{1}-w\right| & =\left|\pi\left(y_{1}\right)-\pi(w)\right| \\
& \leq\left|\pi\left(y_{1}\right)-\pi\left(y_{2}\right)\right|+\left|\pi\left(y_{2}\right)-\pi(w)\right| \\
& \leq 2\left|\pi\left(y_{1}\right)-\pi\left(y_{2}\right)\right| \\
& \leq 2\left|y_{1}-y_{2}\right| .
\end{aligned}
$$

Since $\gamma_{\epsilon_{1}}$ satisfies the 2-point condition (2.1), there exists a subarc $\tau \subset \gamma_{\epsilon_{1}}$ joining $\pi(w)$ to $\pi\left(y_{1}\right)$ and having

$$
\operatorname{diam} \tau_{1} \leq C\left|\pi(w)-\pi\left(y_{1}\right)\right| \leq C\left|w-y_{1}\right| \leq 2 C\left|y_{1}-y_{2}\right| .
$$

Let $\sigma_{1}$ be the lift of $\tau$ and $\sigma_{2}$ be the lift of the line segment $\left[\pi(w), \pi\left(y_{2}\right)\right]$ on $\Sigma(\Omega, \varphi)^{+}$ respectively. Since $\varphi$ is increasing,

$$
\operatorname{diam} \sigma_{2} \leq \varphi\left(\epsilon_{2}\right)-\varphi\left(\epsilon_{1}\right)+\epsilon_{2}-\epsilon_{1} \leq 2\left|y_{2}-w\right| \leq 4\left|y_{1}-y_{2}\right| .
$$

Then $\sigma=\sigma_{1} \cup \sigma_{2}$ is an arc in $\Sigma(\Omega, \varphi)$ that connects $y_{1}$ to $y_{2}$ and has $\operatorname{diam} \sigma \leq$ $(2 C+4)\left|y_{1}-y_{2}\right|$.

Case 3. Suppose that $y_{1}$ and $y_{2}$ are in two different half-spaces. Consider, for instance, that $y_{1} \in \Sigma(\Omega, \varphi)^{+}$and $y_{2} \in \Sigma(\Omega, \varphi)^{-}$and that $\pi\left(y_{1}\right) \in \gamma_{\epsilon_{1}}$ and $\pi\left(y_{2}\right) \in$ $\gamma_{\epsilon_{2}}$. Then $\left|y_{1}-y_{2}\right| \geq \varphi\left(\epsilon_{1}\right)+\varphi\left(\epsilon_{2}\right)$.

Take $v_{1}, v_{2} \in \Gamma$ such that $\left|\pi\left(y_{1}\right)-v_{1}\right|=\epsilon_{1}$ and $\left|\pi\left(y_{2}\right)-v_{2}\right|=\epsilon_{2}$. Denote by $\sigma_{1}$ the lift of the segment $\left[\pi\left(y_{1}\right), v_{1}\right]$ on $\Sigma(\Omega, \varphi)^{+}$and by $\sigma_{2}$ the lift of the segment $\left[\pi\left(y_{2}\right), v_{2}\right]$ on $\Sigma(\Omega, \varphi)^{-}$, respectively. The assumptions on $\varphi$ in the statement of the lemma yield

$$
\operatorname{diam} \sigma_{i} \leq\left|y_{i}-v_{i}\right| \leq \epsilon_{i}+\varphi\left(\epsilon_{i}\right) \leq(1+1 / M) \varphi\left(\epsilon_{1}\right) \leq(1+1 / M)\left|y_{1}-y_{2}\right|,
$$


for $i=1$ and 2. Let $\sigma_{3}$ be a subarc of $\Gamma$ joining $v_{1}, v_{2}$ and having diam $\sigma_{3} \leq$ $C\left|v_{1}-v_{2}\right|$. It follows that

$$
\operatorname{diam} \sigma_{3} \leq C\left(\left|\pi\left(y_{1}\right)-\pi\left(y_{2}\right)\right|+\epsilon_{1}+\epsilon_{2}\right) \leq C(2+1 / M)\left|y_{1}-y_{2}\right| .
$$

The path $\sigma=\sigma_{1} \cup \sigma_{2} \cup \sigma_{3}$ joins $y_{1}, y_{2}$ in $\Sigma(\Omega, \varphi)$ and has

$$
\operatorname{diam} \sigma \leq(2+2 / M)(C+1)\left|y_{1}-y_{2}\right| \text {. }
$$

This proves the claim and Part I.

Part II. The $\mathrm{LLC}_{2}$ property.

We claim that there exists $\lambda>1$ such that, for any $x \in \Sigma(\Omega, \varphi), r>0$ and $y_{1}, y_{2} \in \Sigma(\Omega, \varphi) \backslash B^{3}(x, r)$, there exists a continuum $E \subset \Sigma(\Omega, \varphi) \backslash B^{3}(x, r / \lambda)$ that contains $y_{1}$ and $y_{2}$. Since $\Sigma(\Omega, \varphi) \backslash B^{3}(x, r)$ is non-empty,

$$
r<\operatorname{diam} \Sigma(\Omega, \varphi) \leq \operatorname{diam} \Gamma+2 \varphi(\operatorname{diam} \Gamma) .
$$

To verify the claim, it suffices to show the existence of $\lambda_{0}>1$ when

$$
0<r \leq r_{0}=\min \left\{\epsilon_{0} / 3, \varphi\left(\epsilon_{0} / 3\right)\right\} .
$$

For then, when $r>r_{0}$, points $y_{1}$ and $y_{2}$ are in $\Sigma(\Omega, \varphi) \backslash B^{3}\left(x, r_{0}\right)$, and therefore are contained in a continuum $E$ in $\Sigma(\Omega, \varphi) \backslash B^{3}\left(x, r_{0} / \lambda_{0}\right)$, which in turn is contained in $\Sigma(\Omega, \varphi) \backslash B^{3}\left(x, r /\left(C_{0} \lambda_{0}\right)\right)$, where

$$
C_{0}=\frac{\operatorname{diam} \Gamma+2 \varphi(\operatorname{diam} \Gamma)}{r_{0}} .
$$

We observe that projection $\pi(z)$ of a point $z$ in $\Sigma(\Omega, \varphi) \backslash B^{3}(x, r)$ need not be in $\bar{\Omega} \backslash B^{2}(\pi(x), r)$; this fact complicates the argument below.

Since $0<r<\epsilon_{0} / 3$, the following two cases suffice.

Case 1. Suppose that $\Sigma(\Omega, \varphi) \cap B^{3}(x, r) \subset \overline{\Delta_{\epsilon_{0} / 3}^{+}} \cup \overline{\Delta_{\epsilon_{0} / 3}^{-}}$. Because $\varphi$ is increasing and $0<r \leq \varphi\left(\epsilon_{0} / 3\right), B^{3}(x, r)$ intersects only one of $\overline{\Delta_{\epsilon_{0} / 3}^{+}}$and $\overline{\Delta_{\epsilon_{0} / 3}^{-}}$; assume that $\Sigma(\Omega, \varphi) \cap B^{3}(x, r) \subset \overline{\Delta_{\epsilon_{0} / 3}^{+}}$. Since $\varphi$ is $L$-Lipschitz in $\left[\epsilon_{0} / 3, \infty\right), \Sigma(\Omega, \varphi) \cap B^{3}(x, r)$ contains lift

$$
G=\left\{(w, \varphi(\operatorname{dist}(w, \Gamma))): y \in B^{2}(\pi(x), c(L) r)\right\}
$$

of the disk $B^{2}(\pi(x), c(L) r)$ on $\overline{\Delta_{\epsilon_{0} / 3}^{+}}$, for some $0<c(L)<1$ independent of the point $x$. Then $\Sigma(\Omega, \varphi) \backslash G$ is a continuum in $\Sigma(\Omega, \varphi) \backslash B^{3}(x, c(L) r)$ that contains $y_{1}, y_{2}$.

Case 2. Suppose that $B^{3}(x, r) \cap\left(\Delta_{\epsilon_{0}}^{+} \cup \Delta_{\epsilon_{0}}^{-}\right)=\emptyset$. Given $y_{1}, y_{2} \in \Sigma(\Omega, \varphi) \backslash B^{3}(x, r)$, we consider the following subcases.

(i) Both $y_{1}, y_{2} \in \Delta_{\epsilon_{0}}^{+}$or both $y_{1}, y_{2} \in \Delta_{\epsilon_{0}}^{-}$. There is nothing to prove because either $\Delta_{\epsilon_{0}}^{+}$or $\Delta_{\epsilon_{0}}^{-}$is a continuum exterior to $B^{3}(x, r)$.

(ii) Both $y_{1}, y_{2} \in \Sigma(\Omega, \varphi) \backslash\left(\Delta_{\epsilon_{0}}^{+} \cup \Delta_{\epsilon_{0}}^{-}\right)$. We assume further that $y_{1} \in \Sigma(\Omega, \varphi)^{+} \backslash$ $\Delta_{\epsilon_{0}}^{+}$and $y_{2} \in \Sigma(\Omega, \varphi)^{-} \backslash \Delta_{\epsilon_{0}}^{-}$; other possibilities can be treated similarly. Assume also that $\pi\left(y_{1}\right)$ is on $\gamma_{\epsilon_{1}}$ and $\pi\left(y_{2}\right)$ is on $\gamma_{\epsilon_{2}}$ for some $\epsilon_{1}, \epsilon_{2} \in\left[0, \epsilon_{0}\right]$.

Fix as we may a half-line in $\mathbb{R}^{2}$ starting at a point $x_{0} \in \Delta_{\epsilon_{0}}$ that does not intersect $B^{2}(\pi(x), r)$. Let $v$ be the point on $l \cap \Gamma$ closest to $x_{0}$ and $v_{i}$ be the point on $l \cap \gamma_{\epsilon_{i}}$ closest to $x_{0}$ for $i=1$ or 2 . Note that $v, v_{1}, v_{2}$ are contained in a line segment entirely in $\bar{\Omega}$. Denote by $w_{1}=\left(v_{1}, \varphi\left(\epsilon_{1}\right)\right)$ and $w_{2}=\left(v_{2},-\varphi\left(\epsilon_{2}\right)\right)$ the lifts of $v_{1}$ and $v_{2}$ on $\Sigma(\Omega, \varphi)^{+}$and $\Sigma(\Omega, \varphi)^{-}$, respectively. 
Let $H$ be the hyperplane $\mathbb{R}^{2} \times\left\{\varphi\left(\epsilon_{1}\right)\right\}$ in $\mathbb{R}^{3}$. Then $\tau=\Sigma(\Omega, \varphi) \cap H$, which is the lift $\gamma_{\epsilon_{1}}^{+}$, is a $K$-quasicircle on $H$ that contains $y_{1}$ and $w_{1}$. Assume for a moment that $B^{3}(x, r) \cap H$ is non-empty, so it is a disk $B^{2}(\pi(x), \rho) \times\left\{\varphi\left(\epsilon_{1}\right)\right\}$ with $0<\rho \leq r$. By Remark [5.2 there exists $\lambda_{1}>1$ depending only on $K$ and a subarc $\sigma_{1}$ of $\tau \backslash\left(B^{2}\left(x_{1}, \rho / \lambda_{1}\right) \times\left\{\varphi\left(\epsilon_{1}\right)\right\}\right)$ that connects $y_{1}$ to $w_{1}$. By elementary geometry, the curve $\sigma_{1}$ is in fact contained in $\Sigma(\Omega, \varphi) \backslash B^{3}\left(x, r / \lambda_{1}\right)$. When $B^{3}(x, r) \cap H$ is empty, a curve $\sigma_{1}$ with the required properties exists trivially. Similarly, $y_{2}, w_{2}$ can be joined by an $\operatorname{arc} \sigma_{2} \subset \Sigma(\Omega, \varphi) \backslash B^{3}\left(x, r / \lambda_{1}\right)$ on the plane $\mathbb{R}^{2} \times\left\{-\varphi\left(\epsilon_{2}\right)\right\}$. Finally the union $\sigma_{3}=\left[v, v_{1}\right]^{+} \cup\left[v, v_{2}\right]^{-}$of the lifts of segments $\left[v, v_{1}\right]$ and $\left[v, v_{2}\right]$ connects $w_{1}$ to $v$, then to $w_{2}$ in $\Sigma(\Omega, \varphi)$. Then, $y_{1}, y_{2}$ can be joined in $\Sigma(\Omega, \varphi) \backslash B^{3}\left(x, r / \lambda_{2}\right)$ by the curve $\sigma=\sigma_{1} \cup \sigma_{2} \cup \sigma_{3}$.

(iii) $y_{1} \in \Sigma(\Omega, \varphi) \backslash\left(\Delta_{\epsilon_{0}}^{+} \cup \Delta_{\epsilon_{0}}^{-}\right)$and $y_{2} \in \Delta_{\epsilon_{0}}^{+} \cup \Delta_{\epsilon_{0}}^{-}$. We consider only the case when $y_{1} \in \Sigma(\Omega, \varphi)^{+} \backslash \Delta_{\epsilon_{0}}^{+}$and $y_{2} \in \Delta_{\epsilon_{0}}^{-}$. Proceed as in (ii) to obtain a half-line $l$, points $x_{0}, v, v_{1}$ and $w_{1}$, and a curve $\sigma_{1}$ connecting $y_{1}$ to $w_{1}$. We next take $v_{0}$ to be the point on $l \cap \gamma_{\epsilon_{0}}$ closest to $x_{0}$ and $w_{0}=\left(v_{0},-\varphi\left(\epsilon_{0}\right)\right)$ to be its lift on $\Sigma(\Omega, \varphi)^{-}$. Join $y_{2}$ to $w_{0}$ by any curve $\sigma_{2}^{\prime}$ contained in $\overline{\Delta_{\epsilon_{0}}^{-}}$. Then proceed as in subcase (ii) to define a curve $\sigma_{3}^{\prime}$ in $\Sigma(\Omega, \varphi)$ connecting $w_{1}$ to $v$, then to $w_{0}$ and having projection $\pi\left(\sigma_{3}^{\prime}\right)$ in $l$. The curve $\sigma_{1} \cup \sigma_{2}^{\prime} \cup \sigma_{3}^{\prime}$ has the desired properties.

(iv) Either $y_{1} \in \Delta_{\epsilon_{0}}^{+}$and $y_{2} \in \Delta_{\epsilon_{0}}^{-}$or $y_{1} \in \Delta_{\epsilon_{0}}^{-}$and $y_{2} \in \Delta_{\epsilon_{0}}^{+}$. We assume the former and fix a half-line $l \subset \mathbb{R}^{2}$, points $v_{0}, w_{0}$ and a curve $\sigma_{2}^{\prime}$ as in (iii). Let $u_{0}=\left(u_{0}, \varphi\left(\epsilon_{0}\right)\right)$ be the lift of $v_{0}$ on $\Sigma(\Omega, \varphi)^{+}$, and let $\sigma_{1}^{\prime}$ be any curve in $\overline{\Delta_{\epsilon_{0}}^{+}}$joining $y_{1}$ to $u_{0}$. Finally, as in the previous cases, choose a curve $\sigma_{3}^{\prime \prime} \subset \Sigma(\Omega, \varphi)$ joining $w_{0}$ to $v$, then to $u_{0}$ and having projection $\pi\left(\sigma_{3}^{\prime \prime}\right)$ in $l$. The curve $\sigma_{1}^{\prime} \cup \sigma_{2}^{\prime} \cup \sigma_{3}^{\prime \prime}$ has the properties required.

5.2. Necessary conditions for $\Sigma(\Omega, \varphi)$ to be LLC. We prove the necessity in Proposition 5.1.

Lemma 5.4. Suppose that $\Omega$ is a Jordan domain and that $\Sigma(\Omega, \varphi)$ is $L L C$ for every $\varphi \in \mathcal{F}$. Then $\Omega$ has the $L Q C$ property.

We show first in Lemma 5.5 that if $\Sigma(\Omega, \varphi)$ is $\lambda-\mathrm{LLC}_{1}$ for a gauge $\varphi$ having rapid growth near 0 , then $\gamma_{\epsilon}$ has the level Jordan curve property. We show next in Lemma 5.6 that if $\Gamma$ is a quasicircle and $\Sigma(\Omega, \varphi)$ is $\lambda-\mathrm{LLC}_{1}$ for a gauge $\varphi$ having rapid growth near 0 , then $\Omega$ satisfies the LQC property. Since $\Sigma(\Omega, t)$ is LLC, $\Gamma$ must be a quasicircle by Remark 4.5. This completes the proof of Lemma 5.4 .

Lemma 5.5. Let $\Omega$ be a Jordan domain and $\varphi$ be a function in $\mathcal{F}$ whose almost everywhere derivative $\varphi^{\prime}(t) \rightarrow \infty$ as $t \rightarrow 0$. Suppose that $\Sigma(\Omega, \varphi)$ is $\lambda-L L C_{1}$. Then there exists $\epsilon_{0}>0$ depending only on $\lambda, \varphi$ such that the set $\gamma_{\epsilon}$ is a Jordan curve for every $0 \leq \epsilon \leq \epsilon_{0}$.

Proof. It follows from the assumption on $\varphi^{\prime}$ that there exists $\epsilon_{0}>0$ so that

$$
\varphi\left(t_{2}\right)-\varphi\left(t_{1}\right)>6 \lambda\left(t_{2}-t_{1}\right) \text { for any } 0<t_{1} \leq t_{2} \leq \epsilon_{0} .
$$

The proof contains two claims. First we prove that the set $\Delta_{\epsilon}$ is connected for any $0<\epsilon<\epsilon_{0}$, which implies by Lemma 3.5 that $\Delta_{\epsilon}$ is a Jordan domain. Next we show that $\gamma_{\epsilon}=\partial \Delta_{\epsilon}$ whenever $0<\epsilon<\epsilon_{0}$, which, combined with the first claim, states that $\gamma_{\epsilon}$ is a Jordan curve when $\epsilon<\epsilon_{0}$.

Step 1. We claim that the open set $\Delta_{\epsilon}$ is connected for any $0<\epsilon<\epsilon_{0}$. Suppose, to the contrary, that there exists $0<\epsilon<\epsilon_{0}$ such that the open set $\Delta_{\epsilon}$ has at least 
two components $D_{1}$ and $D_{2}$. This would imply, by the continuity of the distance function, that $\Delta_{\epsilon^{\prime}} \cup \gamma_{\epsilon^{\prime}}$ is not connected for some $\epsilon^{\prime} \in\left(\epsilon, \epsilon_{0}\right)$. From Lemma 3.7 it follows that there exist $\delta<\epsilon_{0}$, a component $D$ of $\Delta_{\delta}$, and three distinct collinear points $x_{0} \in \partial D$ and $x_{1}, x_{2} \in \Gamma$ such that

$$
\left|x_{0}-x_{1}\right|=\left|x_{0}-x_{2}\right|=\delta,
$$

and from Lemma 3.5 it follows that $D$ is a Jordan domain. Observe that $D$ is exterior to the disks $\overline{B^{2}}\left(x_{1}, \delta\right)$ and $\overline{B^{2}}\left(x_{2}, \delta\right)$ and that $\Gamma \cap B^{2}\left(x_{0}, \delta\right)=\emptyset$.

Fix a simple arc $\sigma$ in $D \cup\left\{x_{0}\right\}$ with endpoints $x_{0}$ and $w_{0}$. Heuristically, the set

$$
W=\{(x, z): x \in \sigma,|z| \leq \varphi(\operatorname{dist}(x, \Gamma))\}
$$

serves as a tall, wide wall which prevents two nearby points $z_{1}^{+}$and $z_{2}^{+}$on two sides of $W$ to be joined by a short path without piercing $W$. This violates the LLC $_{1}$ property.

To this end, we fix a point $y_{0}$ on $\sigma$ satisfying

$$
\left|y_{0}-x_{0}\right|<\min \left\{\frac{\left|w_{0}-x_{0}\right|}{2}, \frac{\delta}{16 \lambda}\right\} \text {, }
$$

and set $r=\left|y_{0}-x_{0}\right|$. Simple geometric consideration shows that

$$
\operatorname{dist}\left(y_{0}, \overline{B^{2}}\left(x_{i}, \delta\right)\right) \leq \frac{r^{2}}{\delta}<\frac{r}{16 \lambda} .
$$

Set $\rho=\delta-\frac{r}{8 \lambda}$. Since $\operatorname{dist}\left(y_{0}, \Gamma\right)>\delta$, by the continuity of the distance function, we can find points $z_{1} \in\left[y_{0}, x_{1}\right]$ and $z_{2} \in\left[y_{0}, x_{2}\right]$ with $\operatorname{dist}\left(z_{1}, \Gamma\right)=\operatorname{dist}\left(z_{2}, \Gamma\right)=\rho$. Since $\left|y_{0}-z_{i}\right| \geq \operatorname{dist}\left(y_{0}, \Gamma\right)-\operatorname{dist}\left(z_{i}, \Gamma\right)>\delta-\rho=\frac{r}{8 \lambda}$, the point $z_{i}$ is contained in $B^{2}\left(x_{i}, \delta\right)$; thus

$$
\rho \leq\left|z_{i}-x_{i}\right|<\delta
$$

for $i=1,2$. Therefore $z_{1}$ and $z_{2}$ are in two different components of $B^{2}\left(y_{0}, r\right) \backslash \sigma$, and for $i=1,2$,

$$
\left|z_{i}-y_{0}\right|=\left|y_{0}-x_{i}\right|-\left|x_{i}-z_{i}\right|<\delta+\frac{r}{16 \lambda}-\rho=\frac{3 r}{16 \lambda} .
$$

Recall that $z_{1}^{+}$and $z_{2}^{+}$are the lifts of $z_{1}$ and $z_{2}$ on $\Sigma(\Omega, \varphi)^{+}$. Since

$$
\left|z_{1}^{+}-z_{2}^{+}\right|=\left|z_{1}-z_{2}\right| \leq\left|y_{0}-z_{1}\right|+\left|y_{0}-z_{2}\right|<\frac{3 r}{8 \lambda}
$$

points $z_{1}^{+}$and $z_{2}^{+}$are in the ball

$$
B=B^{3}\left(z_{1}^{+}, \frac{3 r}{8 \lambda}\right) .
$$

Since $\Sigma(\Omega, \varphi)$ is $\lambda-\mathrm{LLC}_{1}$, the points $z_{1}^{+}, z_{2}^{+}$are contained in a continuum $E$ in $\lambda B \cap \Sigma(\Omega, \varphi)$, where $\lambda B=B^{3}\left(z_{1}^{+}, \frac{3 r}{8}\right)$. Note that if $u \in \pi(\lambda B)$, then

$$
\left|u-y_{0}\right| \leq\left|u-z_{1}\right|+\left|z_{1}-y_{0}\right| \leq \frac{3 r}{8}+\frac{3 r}{16 \lambda}<r,
$$

which shows that $\pi(E) \subset \pi(\lambda B) \subset B^{2}\left(y_{0}, r\right)$.

Note that for any $w \in E, w=(\pi(w), \varphi(\operatorname{dist}(\pi(w), \Gamma)))$ and

$$
|\varphi(\operatorname{dist}(\pi(w), \Gamma))-\varphi(\rho)|=\left|\varphi(\operatorname{dist}(\pi(w), \Gamma))-\varphi\left(\operatorname{dist}\left(z_{1}, \Gamma\right)\right)\right| \leq\left|w-z_{1}^{+}\right| \leq \frac{3 r}{8},
$$

and on the other hand by (5.1),

$$
|\varphi(\operatorname{dist}(\pi(w), \Gamma))-\varphi(\rho)| \geq 6 \lambda|\operatorname{dist}(\pi(w), \Gamma)-\rho| .
$$


From these estimates it follows that $\operatorname{dist}(\pi(w), \Gamma)<\rho+\frac{r}{16}<\delta$ for any $w \in E$, and as a consequence $\pi(E)$ does not intersect $\sigma$. This leads to a contradiction, because $\pi(E)$ is a continuum containing two points $z_{1}$ and $z_{2}$ lying in two separate components of $B^{2}\left(y_{0}, r\right) \backslash \sigma$.

Step 2. We claim that $\partial \Delta_{\epsilon}=\gamma_{\epsilon}$ for each $\epsilon \in\left(0, \epsilon_{0}\right)$. Suppose the contrary. Pick a number $\epsilon<\epsilon_{0}$ for which $\partial \Delta_{\epsilon} \varsubsetneqq \gamma_{\epsilon}$. Then, by Lemma 3.7 there exist a component $D$ of $\Delta_{\epsilon}$ and collinear points $x_{0} \in \partial D$ and $x_{1}, x_{2} \in \Gamma$ such that

$$
\left|x_{0}-x_{1}\right|=\left|x_{0}-x_{2}\right|=\epsilon .
$$

The argument leading to a contradiction is similar to that in Step 1; we omit the details.

Lemma 5.6. Let $\Omega$ be a Jordan domain interior to a $K$-quasicircle $\Gamma$ and $\varphi$ be a function in $\mathcal{F}$ whose almost everywhere derivative $\varphi^{\prime}(t) \rightarrow \infty$ as $t \rightarrow 0$. If $\Sigma(\Omega, \varphi)$ is $\lambda-L L C_{1}$ for some $\lambda>1$, then $\Omega$ has the $L Q C$ property. In particular, there exist $\epsilon_{0}>0$ depending only on $\lambda, \varphi$ and $K^{\prime}>1$ depending only on $K, \lambda$ such that $\gamma_{\epsilon}$ is a $K^{\prime}$-quasicircle for any $0<\epsilon \leq \epsilon_{0}$.

Proof. Fix $\epsilon_{0}>0$ so that

$$
\varphi^{\prime}(t) \geq 10 \lambda, \quad \text { for almost every } t \in\left(0, \epsilon_{0}\right) .
$$

By Lemma 5.5, the level set $\gamma_{\epsilon}$ is a Jordan curve for any $\epsilon<\epsilon_{0}$.

Since $\Gamma$ is a $K$-quasicircle, there exists $C>1$ depending only on $K$ so that

$$
\operatorname{diam} \Gamma(x, y) \leq C|x-y|, \quad \text { for all } x, y \in \Gamma \text {. }
$$

It suffices to show that for every $\epsilon \in\left(0, \epsilon_{0}\right)$, the curve $\gamma_{\epsilon}$ satisfies the 2-point condition

$$
\operatorname{diam} \gamma_{\epsilon}(x, y) \leq 50 \lambda C|x-y|, \quad \text { for all } x, y \in \gamma_{\epsilon} .
$$

Otherwise, there exist $\epsilon \in\left(0, \epsilon_{0}\right)$ and points $x_{1}, x_{2}, x_{3}, x_{4}$ on $\gamma_{\epsilon}$ in cyclic order such that $x_{2}$ and $x_{4}$ are on two different components of $\gamma_{\epsilon} \backslash\left\{x_{1}, x_{3}\right\}$ and that

$$
\left|x_{2}-x_{1}\right|,\left|x_{4}-x_{1}\right|>25 \lambda C\left|x_{1}-x_{3}\right| .
$$

We claim that

$$
\left|x_{1}-x_{3}\right|<\frac{\epsilon}{6 \lambda} .
$$

For each $i$ fix a point $p_{i}$ on $\Gamma$ that is nearest to $x_{i}$, so $\left|p_{i}-x_{i}\right|=\epsilon$. By Lemma 3.3, the points $p_{1}, p_{2}, p_{3}, p_{4}$ follow the ordering of $x_{1}, x_{2}, x_{3}, x_{4}$. However some of the points $p_{i}$ might coincide. Note that $\left|p_{1}-p_{3}\right| \leq 2 \epsilon+\left|x_{1}-x_{3}\right|$ and from (5.3) that

$$
\begin{aligned}
C\left|p_{1}-p_{3}\right| & \geq \min \left\{\left|p_{1}-p_{2}\right|,\left|p_{1}-p_{4}\right|\right\} \\
& \geq \min \left\{\left|x_{1}-x_{2}\right|,\left|x_{1}-x_{4}\right|\right\}-2 \epsilon \\
& \geq 25 \lambda C\left|x_{1}-x_{3}\right|-2 \epsilon .
\end{aligned}
$$

Hence, $\left|x_{1}-x_{3}\right|<\frac{\epsilon}{6 \lambda}$. Set $d=\left|x_{1}-x_{3}\right|$.

Since $p_{i}$ is a nearest point on $\Gamma$ to $x_{i}$, the intersection $\left[x_{i}, p_{i}\right] \cap \gamma_{\epsilon-d}$ contains a single point $z_{i}$, and let $z_{i}^{+}=\left(z_{i}, \varphi(\epsilon-d)\right)$ be its lift on $\Sigma(\Omega, \varphi)^{+}$. As before, consider a wall

$$
W^{\prime}=\left\{(x, z): x \in \overline{\Delta_{\epsilon}},|z| \leq \varphi(\operatorname{dist}(x, \Gamma))\right\},
$$

and observe that points $z_{1}^{+}$and $z_{2}^{+}$are separated by a thin part of wall $W^{\prime}$. Any path joining $z_{1}^{+}$to $z_{2}^{+}$without piercing $W^{\prime}$ must climb above or go around the wall; therefore such a path has to be long relative to the distance between $z_{1}^{+}$and $z_{2}^{+}$. 
Consider the ball $B=B^{3}\left(z_{1}^{+}, 5 d\right)$. Since $\left|z_{1}-z_{3}\right| \leq\left|z_{1}-x_{1}\right|+\left|x_{1}-x_{3}\right|+$ $\left|x_{3}-z_{3}\right| \leq 3 d$ we have that $z_{3}^{+} \in B$. By the $\lambda-\mathrm{LLC}_{1}$ there is a continuum $E$ in $\lambda B \cap \Sigma(\Omega, \varphi)$ that contains $z_{1}^{+}, z_{3}^{+}$, where $\lambda B=B^{3}\left(z_{1}^{+}, 5 \lambda d\right)$. Note that for any $w \in E, w=(\pi(w), \varphi(\operatorname{dist}(\pi(w), \Gamma)))$ and that

$$
10 \lambda\left|\pi(w)-z_{1}\right| \leq\left|\varphi(\operatorname{dist}(\pi(w), \Gamma))-\varphi\left(\operatorname{dist}\left(z_{1}, \Gamma\right)\right)\right| \leq\left|w-z_{1}^{+}\right|<5 \lambda d .
$$

Hence $\pi(E)$ is contained in the annular region $A=\bar{\Delta}_{\epsilon-2 d} \backslash \Delta_{\epsilon}$ bordered by two Jordan curves $\gamma_{\epsilon-2 d}$ and $\gamma_{\epsilon}$. Since $\pi(E)$ is a continuum in $\pi(\lambda B) \cap \bar{\Omega}$ that contains $z_{1}, z_{3}$, it must intersect both components in $A \backslash\left(\left[x_{2}, p_{2}\right] \cup\left[x_{4}, p_{4}\right]\right)$, hence intersects at least one of the two segments $\left[x_{2}, p_{2}\right]$ and $\left[x_{4}, p_{4}\right]$. From this it follows that

$$
\begin{aligned}
\operatorname{diam}(\pi(E)) & \geq \min \left\{\left|x_{1}-x_{2}\right|,\left|x_{1}-x_{4}\right|\right\}-\left|x_{1}-z_{1}\right|-\left|x_{3}-z_{3}\right| \\
& \geq 25 \lambda C d-12 d \\
& >13 \lambda d .
\end{aligned}
$$

On the other hand, since $E \subset \lambda B$, diam $\pi(E) \leq 13 \lambda d$, which is a contradiction. Therefore (5.4) must hold.

\section{QUASISYMMETRIC SPHERES OVER QUASIDISKS}

First, we give the proofs of Theorem 1.2 and Corollary 1.4

Proof of Theorem 1.2. To prove the sufficiency, we apply Theorem 2.2 of Bonk and Kleiner. It has been shown, in Proposition 5.1, that if $\Omega$ has the LQC property, then $\Sigma(\Omega, \varphi)$ is LLC for all $\varphi \in \mathcal{F}$. Proposition 6.2 in Section 6.2 below asserts that if $\Omega$ has the LCA property, then $\Sigma(\Omega, \varphi)$ is Ahlfors 2-regular for every $\varphi \in \mathcal{F}$. The sufficiency follows by Theorem 2.2 .

It has been shown in Proposition 5.1 that if $\Sigma(\Omega, \varphi)$ is LLC for all $\varphi \in \mathcal{F}$, then $\Omega$ must have the LQC property; in particular, $\partial \Omega$ is a quasicircle. Proposition 6.3 in Section 6.3 states that if $\partial \Omega$ is a quasicircle but not a chord-arc curve, then there exists a gauge function $\varphi \in \mathcal{F}$, necessarily depending on $\partial \Omega$, so that the associated double-dome-like surface $\Sigma(\Omega, \varphi)$ fails to be quasisymmetric to $\mathbb{S}^{2}$. From these the necessity of the theorem follows.

Proof of Corollary 1.4. Since $\partial \Omega$ is a chord-arc curve with flatness $\zeta_{\partial \Omega}<1 / 2, \Omega$ has the LCA property by Theorem 1.3 . Therefore, by Theorem $1.2, \Sigma(\Omega, \varphi)$ is a quasisymmetric sphere for every $\varphi \in \mathcal{F}$. On the other hand, there exists a domain $\Omega$ whose boundary is a chord-arc curve with flatness $\zeta_{\partial \Omega}=1 / 2$ and which does not satisfy the LQC property; see [24, Remark 5.2] for an example. By Lemma 5.6. $\Sigma\left(\Omega, t^{\alpha}\right)$ is not quasisymmetric to $\mathbb{S}^{2}$ for any $\alpha \in(0,1)$.

The remaining part of this section is devoted to the proofs of Proposition 6.2 and Proposition 6.3.

6.1. Square pieces on $\Sigma(\Omega, \varphi)$ with the assumption of LQC on $\Omega$. Assume that a Jordan domain $\Omega$ has the LQC property: there exist $C_{0}>1$ and $\epsilon_{0}>0$ such that for any $0 \leq \epsilon \leq \epsilon_{0}$,

$$
\operatorname{diam} \gamma_{\epsilon}(x, y) \leq C_{0}|x-y|, \quad \text { for all } x, y \in \gamma_{\epsilon} .
$$

Unless otherwise mentioned, constants and comparison ratios in $\simeq$ and $\lesssim$ in this subsection depend at most on $C_{0}, \epsilon_{0}$ and $\operatorname{diam} \Omega$. 
6.1.1. Quadrilaterals in $\Omega$. A quadruple $\left\langle x_{1}, y_{1}, x_{2}, y_{2}\right\rangle$ of (distinct) points in $\bar{\Omega}$ is said to be admissible if

(i) $x_{1}, y_{1} \in \gamma_{t_{1}}$ and $x_{2}, y_{2} \in \gamma_{t_{2}}$ for some $0 \leq t_{2}<t_{1} \leq \epsilon_{0}$,

(ii) $\left|x_{1}-x_{2}\right|=\operatorname{dist}\left(x_{1}, \gamma_{t_{2}}\right)=\left|y_{1}-y_{2}\right|=\operatorname{dist}\left(y_{1}, \gamma_{t_{2}}\right)=t_{1}-t_{2}$,

(iii) $t_{1}-t_{2} \leq\left|x_{1}-y_{1}\right| / 3 \leq \operatorname{diam} \Omega /\left(10 C_{0}\right)$.

Note from the non-crossing and the monotonicity properties about the distance functions stated in Section 3 that the segments $\left[x_{1}, x_{2}\right]$ and $\left[y_{1}, y_{2}\right]$ do not meet and that arcs $\gamma_{t_{1}}\left(x_{1}, y_{1}\right)$ and $\gamma_{t_{2}}\left(x_{2}, y_{2}\right)$ intersect $\left[x_{1}, x_{2}\right] \cup\left[y_{1}, y_{2}\right]$ only at their endpoints. Denote by $Q\left(x_{1}, y_{1}, x_{2}, y_{2}\right)$ the quadrilateral whose boundary is the Jordan curve $\left[x_{1}, x_{2}\right] \cup\left[y_{1}, y_{2}\right] \cup \gamma_{t_{1}}\left(x_{1}, y_{1}\right) \cup \gamma_{t_{2}}\left(x_{2}, y_{2}\right)$.

Similarly, every $\gamma_{t}, t_{2} \leq t \leq t_{1}$ intersects the segment $\left[x_{1}, x_{2}\right]$ (resp. $\left[y_{1}, y_{2}\right]$ ) at precisely one point, which we call $x^{t}$ (resp. $\left.y^{t}\right)$, and the arc $\gamma_{t}\left(x^{t}, y^{t}\right)$ is contained entirely in $Q\left(x_{1}, y_{1}, x_{2}, y_{2}\right)$. Note from (iii) and the LQC property that

$$
Q\left(x_{1}, y_{1}, x_{2}, y_{2}\right) \text { contains a disk } B^{2}\left(z,\left(t_{1}-t_{2}\right) / 4\right)
$$

centered on $\gamma_{\left(t_{1}+t_{2}\right) / 2}$ and that

$$
\operatorname{diam} \gamma_{t}\left(x^{t}, y^{t}\right) \simeq\left|x_{1}-y_{1}\right|
$$

and

for all $t_{2} \leq t \leq t_{1}$.

$$
\operatorname{diam} Q\left(x_{1}, y_{1}, x_{2}, y_{2}\right) \simeq\left|x_{1}-y_{1}\right|
$$

6.1.2. Square pieces. Let $\varphi$ be a function in $\mathcal{F}$. A quadruple $\left\langle x_{1}, y_{1}, x_{2}, y_{2}\right\rangle$ of points in $\Sigma(\Omega, \varphi)^{+}$is said to be admissible if its projection quadruple $\left\langle\pi\left(x_{1}\right), \pi\left(y_{1}\right), \pi\left(x_{2}\right)\right.$, $\left.\pi\left(y_{2}\right)\right\rangle$ is admissible in $\Omega$. In this case denote by $D=D\left(x_{1}, y_{1}, x_{2}, y_{2}\right)$ the lift of $Q\left(\pi\left(x_{1}\right), \pi\left(y_{1}\right), \pi\left(x_{2}\right), \pi\left(y_{2}\right)\right)$ on the surface $\Sigma(\Omega, \varphi)^{+}$, i.e.,

$$
D\left(x_{1}, y_{1}, x_{2}, y_{2}\right)=\left\{(w, \varphi(\operatorname{dist}(w, \partial \Omega))): w \in Q\left(\pi\left(x_{1}\right), \pi\left(y_{1}\right), \pi\left(x_{2}\right), \pi\left(y_{2}\right)\right)\right\} .
$$

By (i) and (ii),

$$
\left|x_{1}-y_{1}\right|=\left|\pi\left(x_{1}\right)-\pi\left(y_{1}\right)\right|,\left|x_{2}-y_{2}\right|=\left|\pi\left(x_{2}\right)-\pi\left(y_{2}\right)\right| .
$$

If $\left\langle x_{1}, y_{1}, x_{2}, y_{2}\right\rangle$ is admissible in $\Sigma(\Omega, \varphi)^{+}$and satisfies, in addition,

(iv) $\quad \frac{1}{20 C_{0}}\left|x_{1}-y_{1}\right| \leq t_{1}-t_{2}+\varphi\left(t_{1}\right)-\varphi\left(t_{2}\right) \leq \frac{1}{3}\left|x_{1}-y_{1}\right|$,

we say $\left\langle x_{1}, y_{1}, x_{2}, y_{2}\right\rangle$ is admissible for a square piece and call $D\left(x_{1}, y_{1}, x_{2}, y_{2}\right)$ a square piece on $\Sigma(\Omega, \varphi)^{+}$. (Square pieces on $\Sigma(\Omega, \varphi)^{-}$can be defined analogously.) Note from the monotonicity of $\varphi$ that

$$
\left|y_{1}-y_{2}\right|=\left|x_{1}-x_{2}\right| \leq t_{1}-t_{2}+\varphi\left(t_{1}\right)-\varphi\left(t_{2}\right) \leq \sqrt{2}\left|x_{1}-x_{2}\right| .
$$

Also (iv) and the LQC property of $\Omega$ yield that the diameter of a square piece $D\left(x_{1}, y_{1}, x_{2}, y_{2}\right)$ is comparable to $\left|x_{1}-y_{1}\right|$ :

$C_{1}^{-1}\left(t_{1}-t_{2}+\varphi\left(t_{1}\right)-\varphi\left(t_{2}\right)\right) \leq \operatorname{diam} D\left(x_{1}, y_{1}, x_{2}, y_{2}\right) \leq C_{1}\left(t_{1}-t_{2}+\varphi\left(t_{1}\right)-\varphi\left(t_{2}\right)\right)$ for some constant $C_{1}>1$.

Fix a point $z=z\left(x_{1}, y_{1}, x_{2}, y_{2}\right)$, called a rough center of the square piece $D\left(x_{1}, y_{1}, x_{2}, y_{2}\right)$, as follows. First, let $t_{3}$ be the unique number in $\left[t_{2}, t_{1}\right]$ satisfying

$$
t_{3}-t_{2}+\varphi\left(t_{3}\right)-\varphi\left(t_{2}\right)=\frac{1}{2}\left(t_{1}-t_{2}+\varphi\left(t_{1}\right)-\varphi\left(t_{2}\right)\right),
$$

and $\sigma$ be the lift of

$$
\gamma_{t_{3}} \cap Q\left(\pi\left(x_{1}\right), \pi\left(y_{1}\right), \pi\left(x_{2}\right), \pi\left(y_{2}\right)\right)
$$


on $D\left(x_{1}, y_{1}, x_{2}, y_{2}\right) \subset \Sigma(\Omega, \varphi)$. Then, take $z$ to be a point (choices may be plentiful) on the $\operatorname{arc} \sigma$ of equal distance to both endpoints of $\sigma$. It is straightforward to check that

$$
|z-w| \simeq \operatorname{diam} D\left(x_{1}, y_{1}, x_{2}, y_{2}\right), \text { for every } w \in \partial D\left(x_{1}, y_{1}, x_{2}, y_{2}\right) .
$$

Remark 6.1. Let $a$ be a point in $\Sigma(\Omega, \varphi)^{+}$with $\operatorname{dist}(\pi(a), \partial \Omega)<\epsilon_{0}$ and let $0<r \leq$ $\min \left\{\epsilon_{0} / 3, \frac{1}{200 C_{0}^{2}} \operatorname{diam} \Omega\right\}$. Then the surface $\Sigma^{+}(\Omega, \varphi) \cap B^{3}(a, r)$ is contained in a square piece $D_{1}$ and contains a square piece $D_{2}$ with diameters comparable to $r$.

To find $D_{1}$, we set

$$
\begin{aligned}
& t_{1}=\max \left\{\operatorname{dist}(x, \partial \Omega): x \in \pi\left(\Sigma(\Omega, \varphi)^{+} \cap \bar{B}^{3}(a, r)\right)\right\}, \\
& t_{2}=\min \left\{\operatorname{dist}(x, \partial \Omega): x \in \pi\left(\Sigma(\Omega, \varphi)^{+} \cap \bar{B}^{3}(a, r)\right)\right\} .
\end{aligned}
$$

By the monotonicity of $\varphi$,

$$
r \leq t_{1}-t_{2}+\varphi\left(t_{1}\right)-\varphi\left(t_{2}\right) \leq 2 \sqrt{2} r .
$$

Fix a point $z_{1} \in \gamma_{t_{1}}$ whose lift $z_{1}^{+}$on $\Sigma(\Omega, \varphi)^{+}$is contained in $\bar{B}^{3}(a, r)$. Choose $x_{1}, y_{1} \in \gamma_{t_{1}}$ such that $\left|x_{1}-z_{1}\right|=\left|y_{1}-z_{1}\right|=8 C_{0} r$. Let $x_{2}$ (resp. $\left.y_{2}\right)$ be a point on $\gamma_{t_{2}}$ that is closest to $x_{1}$ (resp. $y_{1}$ ). By the LQC property the quadruple $\left\langle x_{1}, y_{1}, x_{2}, y_{2}\right\rangle$ is admissible in $\Omega$. We check that the quadrilateral $Q\left(x_{1}, y_{1}, x_{2}, y_{2}\right)$ contains $\pi\left(\Sigma(\Omega, \varphi)^{+} \cap B^{3}(a, r)\right)$ by showing $|w-\pi(a)|>r$ for every $w \in \gamma_{t} \backslash \gamma_{t}\left(x^{t}, y^{t}\right)$ with $t \in\left[t_{2}, t_{1}\right]$. Given $w \in \gamma_{t} \backslash \gamma_{t}\left(x^{t}, y^{t}\right)$ with $t \in\left[t_{2}, t_{1}\right]$, we fix a point $z^{t}$ in $\gamma_{t}\left(x^{t}, y^{t}\right)$ that is closest to $z_{1}$. The arc $\gamma_{t}\left(w, z^{t}\right)$ contains one of the points $x^{t}, y^{t}$; assume that it contains $x^{t}$. Note that

$$
\operatorname{diam} \gamma_{t}\left(w, z^{t}\right) \geq\left|x^{t}-z^{t}\right| \geq\left|x_{1}-z_{1}\right|-\left|x_{1}-x^{t}\right|-\left|z^{t}-z_{1}\right|>4 C_{0} r .
$$

On the other hand, by the LQC property, diam $\gamma_{t}\left(w, w^{t}\right) \leq C_{0}\left|w-z^{t}\right|$. Hence $|w-\pi(a)| \geq\left|w-z^{t}\right|-\left|z^{t}-\pi(a)\right|>r$.

So $Q\left(x_{1}, y_{1}, x_{2}, y_{2}\right)$ lifts to a square piece $D_{1}=D\left(x_{1}^{+}, y_{1}^{+}, x_{2}^{+}, y_{2}^{+}\right)$which contains $\Sigma(\Omega, \varphi)^{+} \cap B^{3}(a, r)$ and, by (6.4) and (6.6), has diameter

$$
\operatorname{diam} D_{1} \leq 2 \sqrt{2} C_{1} r
$$

recall that $x_{1}^{+}, y_{1}^{+}, x_{2}^{+}, y_{2}^{+}$are the lifts of $x_{1}, y_{1}, x_{2}, y_{2}$ on $\Sigma(\Omega, \varphi)^{+}$.

Define a square piece $D_{2}$ associated to $\Sigma(\Omega, \varphi)^{+} \cap B^{3}\left(a, r /\left(100 C_{1}\right)\right)$ following the steps in constructing $D_{1}$ in the previous paragraph; here $C_{1}$ is the constant in (6.4). So

$$
\operatorname{diam} D_{2} \leq \frac{20 \sqrt{2} C_{1}}{100 C_{1}} r<r / 3
$$

Since $D_{2}$ intersects $B^{3}\left(a, \frac{1}{100 C_{1}} r\right)$, it is contained in $\Sigma(\Omega, \varphi)^{+} \cap B^{3}(a, r)$.

6.2. Ahlfors 2-regularity. We prove in this section the following proposition, which, combined with Theorem 2.2 and Lemma [5.3, concludes the sufficiency in Theorem 1.2

Proposition 6.2. Let $\Omega$ be a planar Jordan that satisfies the level chord-arc property. Then $\Sigma(\Omega, \varphi)$ is Ahlfors 2-regular for every $\varphi \in \mathcal{F}$. 
The statement is quantitative. Suppose that $\Omega$ has the LCA property: there exists $c_{0}>1$ such that for every $0 \leq \epsilon \leq \epsilon_{0}$,

$$
\ell\left(\gamma_{\epsilon}(x, y)\right) \leq c_{0}|x-y|, \quad \text { for all } x, y \in \gamma_{\epsilon} .
$$

Suppose also that $\varphi \in \mathcal{F}$ is $L$-Lipschitz in $\left[\epsilon_{0} / 3, \infty\right)$. Then $\Sigma(\Omega, \varphi)$ satisfies (2.2) for some constant $C$ depending only on $c_{0}, \epsilon_{0}, L$ and $\operatorname{diam} \Omega$.

Proof. Recall that $\Delta_{\epsilon_{0} / 3}$ consists of all points in $\Omega$ whose distance from $\partial \Omega$ is greater than $\epsilon_{0} / 3$ and $\Delta_{\epsilon_{0} / 3}^{+}$is its lift on $\Sigma(\Omega, \varphi)^{+}$. Since $\partial \Delta_{\epsilon_{0} / 3}=\gamma_{\epsilon_{0} / 3}$ is a $c_{0}$-chord-arc curve, the domain $\Delta_{\epsilon_{0} / 3}$ is the bi-Lipschitz image of the unit disk. The surface $\Delta_{\epsilon_{0} / 3}^{+}$, which is the graph of an $L$-Lipschitz function on $\Delta_{\epsilon_{0} / 3}$, is Ahlfors 2-regular. Since the surface $\Sigma(\Omega, \varphi)$ is symmetric with respect to $\mathbb{R}^{2} \times\{0\}$, it suffices to verify that

$$
C^{-1} r^{2} \leq \mathcal{H}^{2}\left(B^{3}(a, r) \cap \Sigma(\Omega, \varphi)^{+}\right) \leq C r^{2},
$$

only for those points $a \in \Sigma(\Omega, \varphi)^{+}$whose projection has $\operatorname{dist}(\pi(a), \partial \Omega)<\epsilon_{0}$ and for $0<r \leq \min \left\{\epsilon_{0} / 3, \frac{1}{180 c_{0}^{2}} \operatorname{diam} \Omega\right\}$. This statement follows immediately from Remark 6.1 and the area estimates (6.10) for square pieces below.

6.2.1. Area of square pieces. Let $\varphi \in \mathcal{F}$ and $D\left(x_{1}, y_{1}, x_{2}, y_{2}\right)$ be a square piece on $\Sigma(\Omega, \varphi)^{+}$. We retain all assumptions and notation associated to the definitions of quadrilaterals and square pieces from the previous subsections.

First we observe that, assuming LQC on $\Omega$, the area of a square piece satisfies

$$
\mathcal{H}^{2}\left(D\left(x_{1}, y_{1}, x_{2}, y_{2}\right)\right) \gtrsim\left|x_{1}-y_{1}\right|^{2} .
$$

When $t_{1}-t_{2} \geq \varphi\left(t_{1}\right)-\varphi\left(t_{2}\right)$, we estimate $\mathcal{H}^{2}\left(D\left(x_{1}, y_{1}, x_{2}, y_{2}\right)\right)$ from below by projecting $D\left(x_{1}, y_{1}, x_{2}, y_{2}\right)$ onto the plane $\mathbb{R}^{2}$, then applying (6.2) and (iv). When $t_{1}-t_{2}<\varphi\left(t_{1}\right)-\varphi\left(t_{2}\right)$, the lower estimate follows from (iv), (6.3) and the Fubini Theorem.

Next we claim that, assuming LCA on $\Omega$, the area of a square piece satisfies

$$
C^{-1}\left|x_{1}-y_{1}\right|^{2} \leq \mathcal{H}^{2}\left(D\left(x_{1}, y_{1}, x_{2}, y_{2}\right)\right) \leq C\left|x_{1}-y_{1}\right|^{2},
$$

for some constant $C>1$ depending on $c_{0}, \epsilon_{0}$ and diam $\Omega$.

Note by (iv) and (6.7) that

$$
\ell\left(\gamma_{t}\left(x^{t}, y^{t}\right)\right) \simeq\left|x_{1}-y_{1}\right|,
$$

for all $t \in\left[t_{2}, t_{1}\right]$, recalling $0 \leq t_{2}<t_{1} \leq \epsilon_{0}$. To establish the upper bound, we let $\epsilon \in\left(0, \frac{1}{3}\left|x_{1}-x_{2}\right|\right)$. Since $\varphi$ is monotone, the length of the graph $\sigma=\{(t, \varphi(t)): t \in$ $\left.\left[t_{2}, t_{1}\right]\right\}$ of $\varphi$ over $\left[t_{2}, t_{1}\right]$ is at most $\sqrt{2}\left|x_{1}-x_{2}\right|$. Hence, there is a partition of $\left[t_{2}, t_{1}\right]$,

$$
t_{2}=\tau_{n}<\cdots<\tau_{i+1}<\tau_{i}<\cdots<\tau_{0}=t_{1},
$$

such that length of the graph $\sigma_{i}$ of $\varphi$ over $\left[\tau_{i+1}, \tau_{i}\right]$ satisfies

$$
\epsilon / 4 \leq \ell\left(\sigma_{i}\right) \leq \epsilon / 2 \text {, }
$$

and therefore the number $n$ in the partition has an upper bound

$$
n \leq \frac{\ell(\sigma)}{\epsilon / 4} \leq \frac{\sqrt{2}\left|x_{1}-x_{2}\right|}{\epsilon / 4} \lesssim \frac{\left|x_{1}-y_{1}\right|}{\epsilon} .
$$

We next partition $D\left(x_{1}, y_{1}, x_{2}, y_{2}\right)$ into strips by the lifts $\gamma_{\tau_{i}}^{+}$of the level curves $\gamma_{\tau_{i}}$. It follows from (iv) and (6.11) that each strip can be covered by at most $C_{2}\left|x_{1}-y_{1}\right| / \epsilon$ balls of radius $\epsilon$. Therefore, $D\left(x_{1}, y_{1}, x_{2}, y_{2}\right)$ can be covered by at 
most $C_{3}\left|x_{1}-y_{1}\right|^{2} / \epsilon^{2}$ balls of radius $\epsilon$. This verifies the upper estimate and the claim (6.10).

6.3. Chord-arc curves and Väisälä's method. In this section we show that the chord-arc property of $\Gamma$ is necessary for $\Sigma(\Omega, \varphi)$ to be a quasisymmetric sphere for all $\varphi \in \mathcal{F}$; this claim together with Proposition 5.1 and Proposition 3.2 concludes the necessity part in Theorem 1.2 .

Proposition 6.3. Suppose that $\Omega$ is a quasidisk but $\Gamma=\partial \Omega$ is not a chord-arc curve. Then there exists $\varphi \in \mathcal{F}$ such that $\Sigma(\Omega, \varphi)$ is not quasisymmetric to $\mathbb{S}^{2}$.

The main idea used in the proof is adapted from Väisälä [22].

Proof. In view of Proposition 5.1 we may assume that $\Omega$ has the LQC property with data $\epsilon_{0}$ and $C_{0}$ as given in (6.1). Constants and comparison ratios in $\simeq$ and $\lesssim$ below depend at most on $\epsilon_{0}, C_{0}$, diam $\Omega$ unless otherwise mentioned.

Since $\Gamma$ is not a chord-arc curve, we may find a sequence $\left\{\Gamma_{n}\right\}_{n \in \mathbb{N}}$ of subarcs of $\Gamma$ such that $\ell\left(\Gamma_{n}\right) / \operatorname{diam} \Gamma_{n} \geq 2 n$ for all $n$ and $\operatorname{diam} \Gamma_{n} \rightarrow 0$ as $n \rightarrow \infty$. For each $n \geq 1$, fix points $x_{n, 0}, x_{n, 1}, \ldots, x_{n, N_{n}}$ on $\Gamma_{n}$, labeled consecutively following their orientation in $\Gamma_{n}$ and with $x_{n, 0}, x_{n, N_{n}}$ representing the endpoints of $\Gamma_{n}$, so that

$$
\sum_{i=1}^{N_{n}}\left|x_{n, i-1}-x_{n, i}\right| \geq n \operatorname{diam} \Gamma_{n}
$$

and that

$$
1 / 2 \leq\left|x_{n, i-1}-x_{n, i}\right| /\left|x_{n, j-1}-x_{n, j}\right| \leq 2, \text { for all } 1 \leq i, j \leq N_{n} .
$$

After passing to a subsequence, we may assume that

$$
d_{n}=\frac{1}{10 C_{0}^{2}} \min _{1 \leq i \leq N_{n}}\left|x_{n, i-1}-x_{n, i}\right|
$$

decrease to zero, as $n \rightarrow \infty$.

Define a homeomorphism $\varphi:[0, \infty) \rightarrow[0, \infty)$ in $\mathcal{F}$ with values

$$
\varphi(0)=0 \text { and } \varphi\left(d_{n}\right)=\operatorname{diam} \Gamma_{n}, \quad \text { for } n \geq 1,
$$

and linear in $\left[d_{1}, \infty\right)$ and in each of the intervals $\left[d_{n}, d_{n-1}\right], n \geq 1$.

We claim that $\Sigma(\Omega, \varphi)$ is not quasisymmetric to $\mathbb{S}^{2}$. Assume the contrary: there exists an $\eta$-quasisymmetric homeomorphism from $\Sigma(\Omega, \varphi)$ onto $\mathbb{S}^{2}$. After post-composing this map with a Möbius transformation, we may find an $\eta$-quasisymmetric embedding $F$ from $\Sigma(\Omega, \varphi)^{+}$to $\mathbb{B}^{2}$.

Now fix $n$. Write $N=N_{n}, d=d_{n}$ and $x_{i}=x_{n, i}$ for simplicity.

Because $\Gamma$ is a quasicircle, by Lemma 3.4 and its proof, we can find for each $i$ a point $w_{i} \in \gamma_{d}$ that satisfies $d \leq\left|w_{i}-x_{i}\right| \leq 3 C_{0} d$. Choose next points $\dot{w}_{i}$ in $\Gamma$ so that $\left|w_{i}-\dot{w}_{i}\right|=\operatorname{dist}\left(w_{i}, \Gamma\right)=d$. Hence $w_{i}, \dot{w}_{i}$ are in $\Gamma \cap B^{2}\left(x_{i}, 4 C_{0} d\right)$. Because $\Gamma$ is a quasicircle satisfying the 2-point condition with the constant $C_{0}$ and the distance between any two consecutive points in $\left\{x_{0}, x_{1}, \ldots, x_{N}\right\}$ is at least $10 C_{0}^{2} d$, sets $\left\{B^{2}\left(x_{i}, 4 C_{0} d\right) \cap \Gamma: 0 \leq i \leq N\right\}$ are mutually disjoint and points $\dot{w}_{0}, \dot{w}_{1}, \ldots, \dot{w}_{N}$ follow the order of $x_{0}, x_{1}, \ldots, x_{N}$ on $\Gamma$ consecutively. Therefore, the segments $\left[w_{i}, \dot{w}_{i}\right]$ are pairwise non-crossing and the points $w_{0}, w_{1}, \ldots, w_{N}$ are also in consecutive order on $\gamma_{d}$. 
Observe again by the LQC property that for every $i=1,2, \ldots, N$

(6.13)

$\operatorname{diam} \Gamma\left(\dot{w}_{i-1}, \dot{w}_{i}\right) \simeq \operatorname{diam} \gamma_{d}\left(w_{i-1}, w_{i}\right) \simeq\left|w_{i-1}-w_{i}\right| \simeq\left|\dot{w}_{i-1}-\dot{w}_{i}\right| \simeq\left|x_{i-1}-x_{i}\right| \simeq d$

and

(6.14)

$\operatorname{diam} \Gamma\left(\dot{w}_{0}, \dot{w}_{N}\right) \simeq \operatorname{diam} \gamma_{d}\left(w_{0}, w_{N}\right) \simeq\left|w_{0}-w_{N}\right| \simeq\left|\dot{w}_{0}-\dot{w}_{N}\right| \simeq\left|x_{0}-x_{N}\right| \simeq \varphi(d)$.

Then

$$
\left|w_{i}^{+}-\dot{w}_{i}\right| \simeq \varphi(d) \text { for } i=0,1, \ldots, N,
$$

recalling that $w_{i}^{+}$is the lift of $w_{i}$ on $\Sigma(\Omega, \varphi)^{+}$.

Note that, by (6.13) and (6.14),$\left\langle w_{0}^{+}, w_{N}^{+}, \dot{w}_{0}, \dot{w}_{N}\right\rangle$ is admissible for a square piece $D=D\left(w_{0}^{+}, w_{N}^{+}, \dot{w}_{0}, \dot{w}_{N}\right)$ in $\Sigma(\Omega, \varphi)^{+}$satisfying diam $D \simeq \varphi(d)$. Here and in the remaining part of the proof, square pieces are understood to satisfy (i), (ii), (iii) and (iv), with the possibility that the constants in (iv) are altered but nevertheless depend at most on $\epsilon_{0}, C_{0}$, diam $\Omega$.

Note also that $\left\langle w_{i-1}, w_{i}, \dot{w}_{i-1}, \dot{w}_{i}\right\rangle$ are admissible in $\Omega$. Let $Q_{i}$ be the quadrilateral bounded by $\Gamma\left(\dot{w}_{i-1}, \dot{w}_{i}\right) \cup\left[w_{i-1}, \dot{w}_{i-1}\right] \cup\left[w_{i}, \dot{w}_{i}\right] \cup \gamma_{d}\left(w_{i-1}, w_{i}\right)$ and $D_{i}=$ $D\left(w_{i-1}^{+}, w_{i}^{+}, \dot{w}_{i-1}^{+}, \dot{w}_{i}^{+}\right)$its lift on $\Gamma(\Omega, \varphi)^{+}$(not necessarily square pieces). Then $\left\{D_{i}: 1 \leq i \leq N\right\}$ have pairwise disjoint interiors.

The square piece $D$ is partitioned into $N$ essentially disjoint tall and narrow strips $D_{i}$, each of which has height in the magnitude $\varphi(d)$ and width in the magnitude $d$. The ratio $N d / \varphi(d)$ of the total width of $D$ to the height is large in view of (6.12). In other words $D$ resembles the product $\sigma \times I$ of a long arc $\sigma$ with a unit interval I. To complete the proof we follow Väisälä's method in [22, Theorem 4.2].

Slice $D_{i}$ by parallel planes $H_{j}=\mathbb{R}^{2} \times\{j d\}$ with $j=0,1, \ldots, m$ and $H_{m+1}=$ $\mathbb{R}^{2} \times\{\varphi(d)\}$, where $m$ is the largest integer less than $\varphi(d) / d-1$. The resulting sets $D_{i, j}, 1 \leq i \leq N$ and $1 \leq j \leq m+1$, are square-like pieces associated to the admissible quadruples $\left\langle v_{i-1, j}, v_{i, j}, v_{i-1, j-1}, v_{i, j-1}\right\rangle$ and have diameter comparable to $d$. Here, $v_{p, q}$ denotes the intersection of the arc $\left[w_{p}, \dot{w}_{p}\right]^{+}$and the plane $H_{q}$.

Let $z_{i, j}$ be a rough center of $D_{i, j}$ defined as in Section 6.1.2. Then by (6.5)

$$
\left|z_{i, j}-x\right| \simeq \operatorname{diam} D_{i, j} \simeq d, \quad \text { for any } x \in \partial D_{i, j} .
$$

Let $u \in \partial D_{i j}$ be a point at which

$$
\left|F\left(z_{i, j}\right)-F(u)\right|=\operatorname{dist}\left(F\left(z_{i, j}\right), \partial F\left(D_{i, j}\right)\right)=r .
$$

Since $\left|v_{i, j-1}-v_{i, j}\right| \simeq\left|v_{i, j}-z_{i, j}\right| \simeq\left|z_{i, j}-u\right|$, it follows from the $\eta$-quasisymmetry of $F$ that $\beta_{i, j}=F\left(v_{i, j-1}\right)-F\left(v_{i, j}\right) \lesssim r$. Hence $\beta_{i, j}^{2} \lesssim \mathcal{H}^{2}\left(F\left(D_{i, j}\right)\right)$. Here and in the rest of the proof, constants may also depend on $\eta$.

Set

$$
\beta=\min \left\{|F(x)-F(y)|: x \in \gamma_{d}\left(w_{0}, w_{N}\right)^{+}, y \in \Gamma\left(\dot{w}_{0}, \dot{w}_{N}\right)\right\} .
$$

By Schwarz inequality,

$$
\beta^{2} \leq\left(\sum_{j=1}^{m+1} \beta_{i, j}\right)^{2} \lesssim(m+1) \mathcal{H}^{2}\left(F\left(D_{i}\right)\right)
$$

for $i=1,2, \ldots, N$. Note from (6.12) that $n \varphi(d) \lesssim N d$ and, by the choice of $m$, that $\varphi(d) / d-2 \leq m<\varphi(d) / d-1$. Summing over $i$ we get

$$
n \beta^{2} \lesssim \mathcal{H}^{2}(F(D)) \text {. }
$$


Let $p \in \gamma_{d}\left(w_{0}, w_{N}\right)^{+}$and $q \in \Gamma\left(\dot{w}_{0}, \dot{w}_{N}\right)$ be the points at which $\beta=|F(p)-F(q)|$ is realized. Then $|x-p| \lesssim \operatorname{diam} D \simeq|p-q|$ for any $x \in D$, which implies by quasisymmetry that $|F(x)-F(p)| \lesssim \beta$. Since $F(D)$ is planar, $\mathcal{H}^{2}(F(D)) \lesssim \beta^{2}$. Hence by (6.16), $n \beta^{2} \lesssim \beta^{2}$.

This is impossible since $\beta$ and the comparison ratio are independent of $n$. The proof is completed.

\section{REFERENCES}

[1] Lars V. Ahlfors, Quasiconformal reflections, Acta Math. 109 (1963), 291-301. MR.0154978 $(27$ \#4921)

[2] A. Beurling and L. Ahlfors, The boundary correspondence under quasiconformal mappings, Acta Math. 96 (1956), 125-142. MR0086869(19,258c)

[3] Christopher J. Bishop, A quasisymmetric surface with no rectifiable curves, Proc. Amer. Math. Soc. 127 (1999), no. 7, 2035-2040, DOI 10.1090/S0002-9939-99-04900-X. MR.1610908 (99j:30023)

[4] Mario Bonk and Bruce Kleiner, Quasisymmetric parametrizations of two-dimensional metric spheres, Invent. Math. 150 (2002), no. 1, 127-183, DOI 10.1007/s00222-002-0233-z. MR.1930885 (2004k:53057)

[5] G. David and T. Toro, Reifenberg flat metric spaces, snowballs, and embeddings, Math. Ann. 315 (1999), no. 4, 641-710, DOI 10.1007/s002080050332. MR1731465 (2001c:49067)

[6] John B. Garnett and Donald E. Marshall, Harmonic measure, New Mathematical Monographs, vol. 2, Cambridge University Press, Cambridge, 2008. Reprint of the 2005 original. MR2450237(2009k:31001)

[7] F. W. Gehring, Rings and quasiconformal mappings in space, Trans. Amer. Math. Soc. 103 (1962), 353-393. MR0139735 (25 \#3166)

[8] F. W. Gehring, Extension of quasiconformal mappings in three space, J. Analyse Math. 14 (1965), 171-182. MR0179354 (31 \#3602)

[9] F. W. Gehring, Quasiconformal mappings of slit domains in three space, J. Math. Mech. 18 (1969), 689-703. MR0245786 (39 \#7092)

[10] Frederick W. Gehring, Characterizations of quasidisks, Quasiconformal geometry and dynamics (Lublin, 1996), Banach Center Publ., vol. 48, Polish Acad. Sci., Warsaw, 1999, pp. 11-41. MR:1709972 (2000g:30014)

[11] F. W. Gehring and J. Väisälä, The coefficients of quasiconformality of domains in space, Acta Math. 114 (1965), 1-70. MR0180674 (31 \#4905)

[12] Juha Heinonen, Lectures on analysis on metric spaces, Universitext, Springer-Verlag, New York, 2001. MR:1800917 (2002c:30028)

[13] David S. Jerison and Carlos E. Kenig, Hardy spaces, $A_{\infty}$, and singular integrals on chord-arc domains, Math. Scand. 50 (1982), no. 2, 221-247. MR672926 (84k:30037)

[14] John L. Lewis and Andrew Vogel, On pseudospheres that are quasispheres, Rev. Mat. Iberoamericana 17 (2001), no. 2, 221-255. MR1891198 (2003b:30029)

[15] Daniel Meyer, Snowballs are quasiballs, Trans. Amer. Math. Soc. 362 (2010), no. 3, 12471300, DOI 10.1090/S0002-9947-09-04635-2. MR2563729 (2011a:30067)

[16] Pekka Pankka and Jang-Mei Wu, Geometry and quasisymmetric parametrization of Semmes spaces, Rev. Mat. Iberoam. 30 (2014), no. 3, 893-960, DOI 10.4171/RMI/802. MR.3254996

[17] Ch. Pommerenke, Boundary behaviour of conformal maps, Grundlehren der Mathematischen Wissenschaften [Fundamental Principles of Mathematical Sciences], vol. 299, Springer-Verlag, Berlin, 1992. MR,1217706 (95b:30008)

[18] Pekka Tukia, Extension of quasisymmetric and Lipschitz embeddings of the real line into the plane, Ann. Acad. Sci. Fenn. Ser. A I Math. 6 (1981), no. 1, 89-94, DOI 10.5186/aasfm.1981.0624. MR639966 (83d:30022)

[19] P. Tukia and J. Väisälä, Quasisymmetric embeddings of metric spaces, Ann. Acad. Sci. Fenn. Ser. A I Math. 5 (1980), no. 1, 97-114, DOI 10.5186/aasfm.1980.0531. MR595180(82g:30038)

[20] P. Tukia and J. Väisälä, Quasiconformal extension from dimension $n$ to $n+1$, Ann. of Math. (2) 115 (1982), no. 2, 331-348, DOI 10.2307/1971394. MR647809 (84i:30030)

[21] Jussi Väisälä, Quasi-Möbius maps, J. Analyse Math. 44 (1984/85), 218-234, DOI 10.1007/BF02790198. MR801295 (87f:30059) 
[22] Jussi Väisälä, Quasisymmetric maps of products of curves into the plane, Rev. Roumaine Math. Pures Appl. 33 (1988), no. 1-2, 147-156. MR948449 (89e:30041)

[23] Jussi Väisälä, Quasiconformal maps of cylindrical domains, Acta Math. 162 (1989), no. 3-4, 201-225, DOI 10.1007/BF02392837. MR989396 (90f:30034)

[24] Vyron Vellis and Jang-Mei Wu, Sets of constant distance from a Jordan curve, Ann. Acad. Sci. Fenn. Math. 39 (2014), no. 1, 211-230, DOI 10.5186/aasfm.2014.3905. MR3186814

[25] M. F. Walker, Linearly locally connected sets and quasiconformal mappings, Ann. Acad. Sci. Fenn. Ser. A I Math. 11 (1986), no. 1, 77-86, DOI 10.5186/aasfm.1986.1118. MR826350 (88c:30025)

Department of Mathematics, University of Illinois, 1409 West Green Street, UrBANA, IlLiNOIS 61820

E-mail address: vellis1@illinois.edu

Current address: Department of Mathematics and Statistics, P. O. Box 35, University of Jyväskylä, FI-40014, Finland

E-mail address: vyron.v.vellis@jyu.fi

Department of Mathematics, University of Illinois, 1409 West Green Street, UrBANA, ILLINOIS 61801

E-mail address: jmwu@illinois.edu 